\title{
Data on Surface-Water Quality and Quantity, Lower Edgewood Creek Basin, Douglas County, Nevada, 1984-85
}

By Richard J. La Camera and Sherwood B. Browning

U.S. GEOLOGICAL SURVEY

Open-File Report 87-565

Prepared in cooperation with the

U.S. FOREST SERVICE and the

TAHOE REGIONAL PLANNING AGENCY

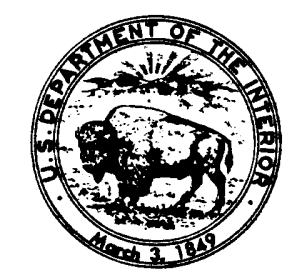

Carson City, Nevada 
DEPARTMENT OF THE INTERIOR

DONALD PAUL HODEL, Secretary

U.S. GEOLOGICAL SURVEY

Dallas L. Peck, Director

For additional information

write to:

\section{U.S. Geological Survey}

Room 227, Federal Building

705 North Plaza Street

Carson City, NV 89701
Copies of this report may be purchased from:

U.S. Geological Survey

Books and Open-File Reports Section

Federal Center

Box 25425

Denver, CO 80225 
ABSTRACT - 1

INTRODUCTION - 1

Purpose and scope - 1

Acknowledgment 3

DESCRIPTION OF THE WATER-QUALITY SAMPLING SITES -

CLIMATOLOGIC AND HYDROLOGIC CONDITIONS DURING THE STUDY PERIOD - 5

Site 1 - 5

Site 2 - 5

Site 3 - 6

Site 4 - 6

DISCUSSION OF THE DATA TABLES - 7

SUMMARY - 8

REFERENCES CITED - 8

DATA ON SURFACE-WATER QUALITY AND QUANTITY - 9

\section{ILLUSTRATION}

Figure 1. Map showing water-quality sampling sites -

TABLES

Table 1. Water-quality and water-quantity data, August 1984-July 1985

2. Selected summary statistics for constituents and characteristics 14

3. Constituent loads - 16 
"Inch-pound" units of measure used in this report may be converted to metric (International System) units by using the following factors:

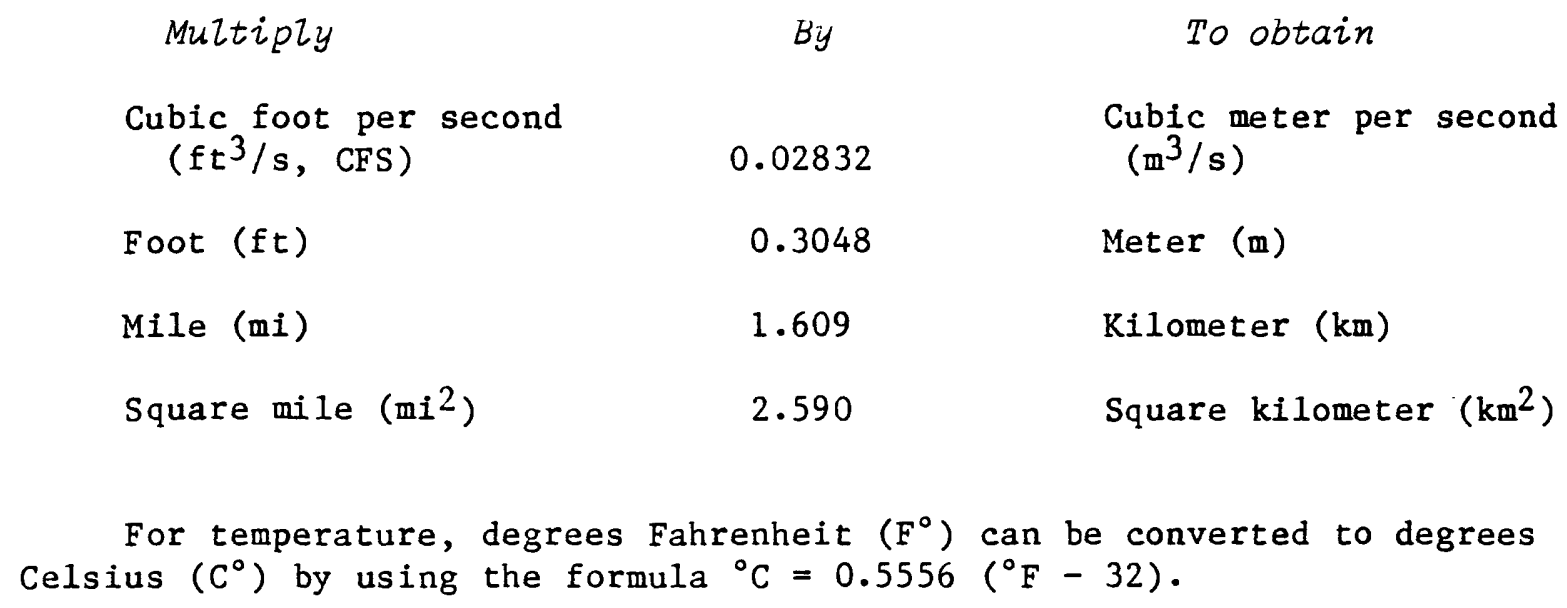

For temperature, degrees Fahrenheit $\left(F^{\circ}\right)$ can be converted to degrees Celsius $\left(\mathrm{C}^{\circ}\right)$ by using the formula ${ }^{\circ} \mathrm{C}=0.5556\left({ }^{\circ} \mathrm{F}-32\right)$. as follows:

Abbreviations for metric water-quality units used in tables 1 and 2 are

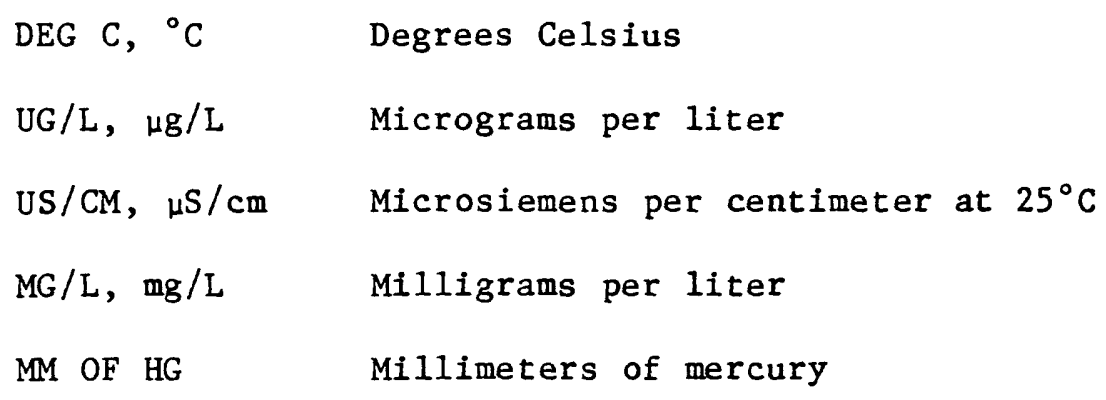


DATA ON SURFACE-WATER QUALITY AND QUANTITY, LOWER EDGEWOOD CREEK BASIN, DOUGLAS COUNTY, NEVADA, 1984-85

By Richard J. La Camera and Sherwood B. Browning

\section{ABSTRACT}

Selected hydrologic data were collected from August 1984 through July 1985 at three sites on the lower part of Edgewood Creek, and at a recently constructed sediment-catchment basin that captures and retains runoff from developed areas in the lower Edgewood Creek drainage.

The data were collected to quantify the discharge of selected constituents (1) downstream from recent and planned watershed-restoration projects and (2) to Lake Tahoe. Contained in this report are the results of quantitative analyses of 39 water samples for: total and dissolved ammonium, organic nitrogen, nitrite, nitrate, phosphorus, and orthophosphorus; suspended sediment; total iron, manganese, and zinc; and dissolved solids. Also included are: field measurements of streamflow, water temperature, specific conductance, $\mathrm{pH}$, and dissolved oxygen; summary statistics (means and standard deviations), and computations of instantaneous loads.

\section{INTRODUCTION}

The U.S. Geological Survey, in cooperation with the U.S. Forest Service and the Tahoe Regional Planning Agency, collected and analyzed selected water-quality and water-quantity data in the lower part of the Edgewood Creek drainage basin (figure 1) between August 1984 and July 1985.

The Edgewood Creek basin is near State Highway 207 and Stateline, Nev., and encompasses approximately 6 square miles of the Lake Tahoe basin. The lands have a slight to moderate erosion potential where soil is exposed, slow to very slow infiltration rates when thoroughly wetted, and moderately high to high runoff potential within the study area (Bailey, 1974).

\section{Purpose and Scope}

The purposes of this report are to: (1) present selected hydrologic data collected from August 1984 through July 1985 at three sites on the lower part of Edgewood Creek and at a recently constructed sedimentcatchment basin, which captures and retains runoff from developed areas in the lower Edgewood Creek Basin; (2) describe conditions under which the data were collected; and ( 3 ) summarize the collected data by using basic statistical techniques. 


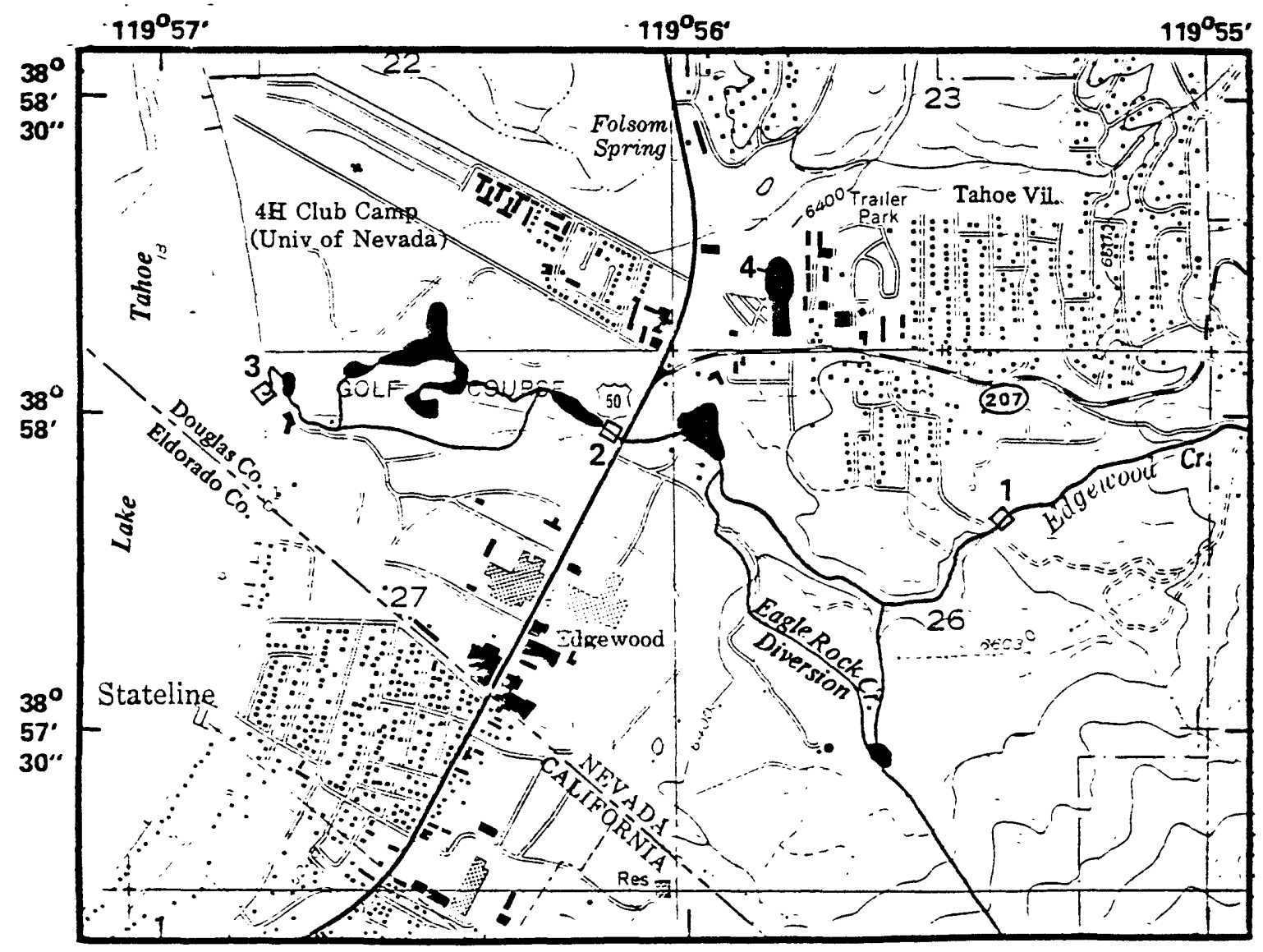

Base trom U.S. Geolorical Survey. South Lake

Tahoe, Callf.-Nev.. 1955.

photorevised 1982
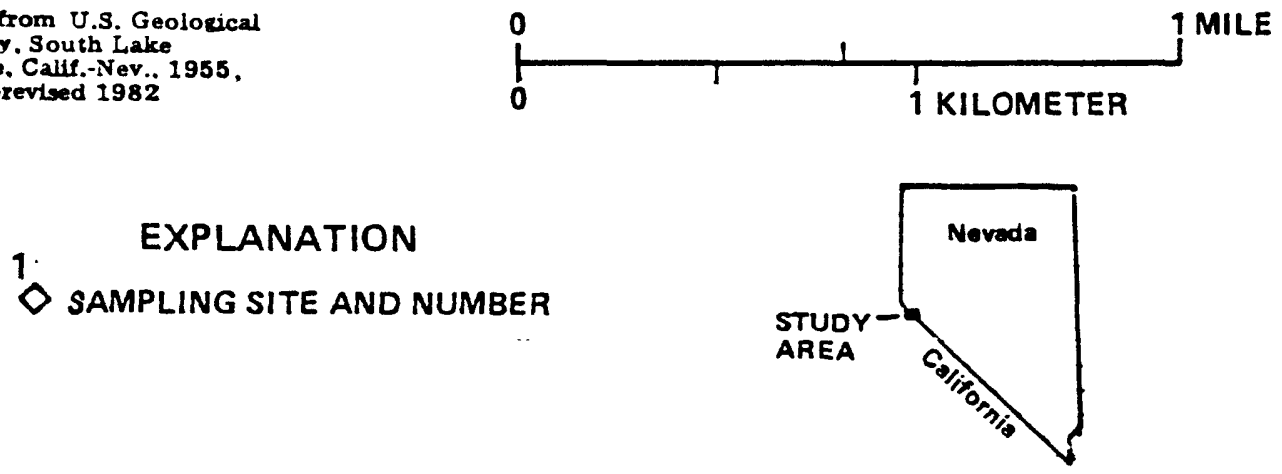

FIGURE 1.-Water-quality sampling sites. 
Water samples were analyzed for: total and dissolved ammonium ${ }^{1}$, ammonium plus organic nitrogen, nitrite, nitrite plus nitrate, phosphorus, and orthophosphorus; suspended sediment; total iron, manganese, and zinc; and dissolved solids. Field measurements included streamflow, water temperature, specific conductance, $\mathrm{pH}$, and dissolved oxygen.

Standard suspended-sediment sampling techniques were used (Guy and Norman, 1970). Sampling frequency at most sites was approximately weekly during the spring, when the greatest quantities of the constituents of interest were expected to be mobilized because of high flows, and occasionally at other times of the year. A total of 39 data sets were collected during the sampling period.

\section{Acknowledgment}

The cooperation of Steve Seibel, Superintendent of the Edgewood Tahoe Golf Course, who allowed access to private property, is greatly appreciated.

\section{DESCRIPTION OF THE WATER-QUALITY SAMPLING SITES}

Locations of the sampling sites are shown in figure 1.

Site 1 (station 10336759), Edgewood Creek near Stateline, Nev., is a Survey stream-gaging station currently operated for streamflow, fluvialsediment, and nutrient information. This site indicates stream quality, including the quantity of transported material, downstream from recent and planned watershed-restoration projects. Samples at site 1 were collected from a footbridge at the gaging station. Any comparisons between data from this site and sites 2 or 3 should also consider ( 1 ) the inflow from the Eagle Rock Creek diversion (fig. 1), for which discharge was only slightly less than that of Edgewood Creek during much of the study period; (2) the intermittent inflow of several small tributaries observed between sites 1 and 2; and (3) the frequent regulation of streamflow by the small reservoir just upstream from U.S. Highway 50 .

Site 2 (station 10336761), Edgewood Creek below U.S. Highway 50 near Stateline, Nev., is immediately upstream from all golf-course ponds and diversions and about 75 feet downstream from U.S. Highway 50. Although the data at this site are extremely limited, comparisons with data from site 3 may provide some insight into possible effects of the golf-course impoundments and diversions on the nature and quantity of materials through that reach of Edgewood Creek. Such comparisons, as well as more frequent sampling to allow more definitive conclusions, were beyond the scope of this study.

1 Throughout the text and in tables 2 and 3 , the term "ammonium" is used to signify the combined concentration of ammonia (the dissolved gas $\mathrm{NH}_{3}$ ) plus ammonium (the ion $\mathrm{NH}_{4}^{+}$), expressed as nitrogen (the element $\mathrm{N}$ ). In table 1 , the terms "ammonia" and " $\mathrm{NH}_{4}$ " signify the same combined concentration. 
Site 3 (station 10336765), Edgewood Creek at Lake Tahoe near Stateline, Nev., is at the mouth of Edgewood Creek, immediately upstream from the shoreline of Lake Tahoe on the Edgewood Golf Course. This site indicates stream quality at the mouth of Edgewood Creek and the quantity of material delivered to Lake Tahoe from the entire Edgewood Creek drainage. Water samples were collected at the mouth of a culvert that carries outflow from the lowermost golf-course pond.

Site 4 (station 103367595), Sediment-catchment basin near Tahoe Village, is a large manmade basin completed in the summer of 1984 approximately $\frac{1}{4}$ mile east of U.S. Highway 50 and just north of State Highway 207 . No outflow from the basin to Edgewood Creek is anticipated; on rare, extreme runoff events, however, the catchment basin could discharge water through an underground catchment drain that crosses underneath State Highway 207 and empties into a small tributary of Edgewood Creek.

Except for the period from March 30 through April 6, 1985, samples were collected at the mouth of the underground storm drain that empties into the catchment basin. During the period March 30-April 6, when access to the storm drain was not possible, the samples were collected from the banks of the catchment basin about 15 feet downstream from the mouth of the storm drain.

The data collected at site 4 must be qualified. Except for samples obtained in July 1985, which were collected in flowing water at the mouth of the storm drain and represent runoff following a thundershower, the data probably represent the quality of water being retained in the catchment basin, rather than that of actual runoff into the catchment basin.

The design of the catchment basin is such that when runoff begins, water flows into the catchment basin from the storm drain. As runoff continues, the water level in the catchment basin rises and water begins to back up into the mouth of the storm drain. During periods of prolonged runoff, such as spring snowmelt, the water level in the catchment basin continues to rise until the mouth of the storm drain is completely submerged. This causes water to back up farther into the storm drain, and results in a greatly reduced velocity of runoff entering the catchment basin.

When samples were collected at the storm-drain mouth, much of the suspended material contained in the actual runoff probably had been deposited in the storm drain due to the reduced velocity there. Additionally, the banks of the newly constructed catchment basin had not yet stabilized, and as the water level in the basin rose, large mats of straw from the bank cover (used to reduce surficial bank erosion) entered the water. The effects of straw on water chemistry in the catchment basin, as well as the effects of localized snowmelt and its associated runoff from the banks of the catchment basin itself, could not be isolated from the urban runoff when standing water was sampled. 


\section{CLIMATOLOGIC AND HYDROLOGIC CONDITIONS DURING THE STUDY PERIOD}

Precipitation during the study period was approximately 80 percent of normal (National Weather Service, oral commun., 1985). As a result, streamflow was somewhat lower than previously measured. In addition, streamflow associated with spring snowmelt occurred earlier than normal and remained high for a shorter period of time than usual, due to a rapid warming in early April.

A brief, generalized summary of weather conditions during the study period is presented below. Air temperatures and snowfall amounts are approximations based on observations nade by the authors at lake level near Edgewood Creek.

Major storms deposited 1 to 2 feet of snow in the area on December 15 , 1984, February 7-8, February 28 to March 7, and March 24-28, 1985. Following the stormy period in late March, maximum air temperatures increased from about $55^{\circ} \mathrm{F}$ to about $75^{\circ} \mathrm{F}$, and much of the snow melted from the higher altitudes by mid-April. In mid-April, a period of unsettled weather brought cooler temperatures (highs ranging from about $40^{\circ} \mathrm{F}$ to about $55^{\circ} \mathrm{F}$ ) until the end of the month, followed by maximum air temperatures of about $60-70^{\circ} \mathrm{F}$ through the month of May. During this period, minor amounts of precipitation fell on about April 17, April 20, and May 10, and the remainder of the s nowpack gradually melted. A minor storm deposited about an inch of wet snow June 1 and 2 , which melted rapidly; no further precipitation fell in June, and temperatures were unseasonably warm, ranging from about $75^{\circ} \mathrm{F}$ to about $85^{\circ} \mathrm{F}$. Heavy thundershowers occurred on July 21,26 , and 27 . More detailed information concerning site-specific sampling conditions are listed below.

\section{Site 1}

On August 21, 1984, water samples were collected during stable flow conditions (at 1100 hours) and just following the peak of a slight rise in streamflow resulting from a thundershower (at 1200). Relatively large diurnal fluctuations in streamflow occurred during the first half of April 1985. Samples on April 4 were collected near the minimum flow for the day. On April 15, samples were collected during a rise in streamflow (at 1445), and at the time of maximum discharge for that day (at 1830). Samples collected on June 4 followed a slight increase in streamflow which occurred 2 days earlier as a result of a minor storm, and some foam was visible on the water surface. The streamflow was relatively stable during times of sample collection for all other dates.

\section{Site 2}

Conditions at times of sample collection were largely unknown at site 2, due to frequent regulation of outflow by the small reservoir above U.S. Highway 50 and the lack of detailed records pertaining to storage or release of water in the reservoir. 
Streamflow had recently been greater prior to sample collection on April 29, 1985, but no obvious sign of large fluctuation in streamflow was apparent at other times of sample collection.

\section{Site 3}

Irrigation on the golf course was observed for the first time on April 15, 1985, and was noted on every visit thereafter. The golf course was fertilized for the first time in 1985 on April 23, but subsequent dates of application are unknown. Storage of water in the small golf-course impoundments was first noticed on April 29, and also continued for the duration of the study. On May 23, a film of pine pollen was visible on the lake surface, and the blue-green algae, Oscillatoria, which thrives in warm, nutrient-rich water (R. J. Hoffman, U.S. Geological Survey, oral commun., 1985), was identified just below the mouth of the storm drain where samples were collected.

\section{Site 4}

On March 30, 1985, water samples were collected following a major snowstorm and subsequent warming. Snow covered the banks of the catchment basin, and muddy ice covered the entire water surface. When samples were collected on April 4, most of the ice had melted but snow still covered the banks of the basin. By April 6, all ice had melted from the water surface and most of the snow had melted from the banks of the catchment basin; the storm-drain mouth was completely submerged by turbid water. A large amount of straw was observed floating on the water surface on April 6 and 15. By April 23, the water level in the catchment basin had dropped to just slightly below the top of the storm-drain mouth, and by May 7 , some grass had begun to establish itself on the banks of the catchment basin. On June 4 (when samples were collected following some probable inflow on June 2), the water appeared more turbid than the last few visits and some pine pollen was visible on the water surface. On July 26, a heavy thundershower lasted approximately 25 minutes, followed by a subsequent light rain for about 10 additional minutes. Samples were collected near the peak inflow to the catchment basin (at 1640 hours, which was about 40 minutes after the beginning of rainfall) and during the flow recession (at 1650). The inflow was extremely turbid, and some organic debris was visible on the water surface. The water also foamed greatly when agitated. Unfortunately, most of the samples collected at 1640 hours were inadvertently destroyed in the laboratory.

A reference point was established at the mouth of the storm drain 0.25 foot below its top. The elevation of the water surface in the catchment basin was determined relative to this point, and the measurements are tabulated below. A negative value indicates that the water surface was below the reference point and a positive value indicates that the water surface was above the reference point. A lower number, therefore, indicates a lesser amount of water retained in the basin. 


\begin{tabular}{lllll}
\hline \multicolumn{1}{c}{ Date } & Feet & \multicolumn{2}{c}{ Date } & Feet \\
\hline Nov. 6, 1984 & -2.8 & May 7, 1985 & -0.9 \\
Jan. 28, 1985 & -3.0 & May 15, 1985 & -1.3 \\
March 30, 1985 & -0.5 (estimated) & May 23, 1985 & -1.8 \\
Apri1 6, 1985 & +1.5 (estimated) & May 28, 1985 & -2.2 \\
Apri1 15, 1985 & +0.5 & June 2, 1985 & -2.3 \\
Apri1 23, 1985 & +0.1 & June 4, 1985 & -2.1 \\
Apri1 29, 1985 & -0.4 & & & \\
\hline
\end{tabular}

\section{DISCUSSION OF THE DATA TABLES}

The raw data collected during the study period are tabulated in table 1. Table 2 tabulates the number of measurements, mean, standard deviation, minimum value, and maximum value for the constituent concentrations and characteristics measured. Note that for site 4, data collected on July 26 have been excluded from the calculations because they represented conditions which were distinctly different from all other conditions.

Table 3 is a tabulation of measured instantaneous and mean loads for the study period for all constituents. The values are rounded to two significant figures. Loads were not calculated for data collected at the sediment-catchment basin because of (1) the limited availability of discharge data and (2) other difficulties discussed above.

Although inconclusive because of the limited data available and short time frame of this study, some observations concerning the discharge of nitrogen and phosphorus can be made on the basis of mean loads listed in table 3. At each of the three Edgewood Creek sites (sites 1-3), about 80 percent of the total nitrogen load is in the form of organic nitrogen, 12 percent is in the form of nitrate nitrogen, 7 percent is in the form of ammonium nitrogen, and 1 percent is in the form of nitrite nitrogen. At the three stream sites, the percentage of total phosphorus load in the form of orthophosphorus varies somewhat with time, but is generally greater at downstream sites 2 and 3 than at upstream site 1 . In addition, the percentage of the total phosphorus load that is present in the dissolved state generally is greater at the two downstream stations than at the upstream site. 


\section{SUMMARY}

Selected hydrologic data were collected from August 1984 through July 1985 at three sites on the lower part of Edgewood Creek and at a recently constructed sediment-catchment basin that captures and retains runoff from developed areas in the lower Edgewood Creek drainage.

The data were collected to quantify the discharge of selected constituents ( 1 ) from recent and planned watershed-restoration areas and (2) to Lake Tahoe. This report contains the results of quantitative analyses of 39 water samples for: total and dissolved ammonium, organic nitrogen, nitrite, nitrate, phosphorus, and or thophosphorus; suspended sedinent; total iron, manganese, and zinc; and dissolved solids. Also included are: field measurements of streamflow, water temperature, specific conductance, $\mathrm{pH}$, and dissolved oxygen; summary statistics (means and standard deviations), and computations of instantaneous loads.

On the basis of mean values, about 80 percent of the total nitrogen load at each of the three Edgewood Creek sites is in the form of organic nitrogen, 12 percent is in the form of nitrate nitrogen, 7 percent is in the form of ammonium nitrogen, and 1 percent is in the form of nitrite nitrogen. The percentage of total phosphorus load in the form of orthophosphorus at the three stream sites varies somewhat with time, but is generally greater at the two downstream sites than at the upstream site. In addition, the percentage of the total phosphorus load that is present in the dissolved state generally is greater at the two downstream sites than at the upstream site.

\section{REFERENCES CITED}

Bailey, R. G., 1974, Land-capability classification of the Lake Tahoe Basin, California-Nevada--a guide for planning: U. S. Forest Service report, $32 \mathrm{p}$.

Gilliom, R. J., and Helsel, D. R., 1984, Estimation of distributional parameters for censored trace-level water-quality data--I. Estimation techniques: U.S. Geological Survey Open-File Report 84-729, 25 p.

Guy, H. P., and Norman, V. W., 1970, Field methods for measurement of fluvial sediment: U.S. Geological Survey Techniques of Water-Resources Investigations, Book 3, Chapter C2, 59 p.

Helsel, D. R., and Gilliom, R. J., 1985, Estimation of distributional parameters for censored trace-level water-quality data--II. Verification and applications: U.S. Geological Survey Open-File Report 85-86, 21 p. 


\section{Data on Surface-Water Quality and Quantity}




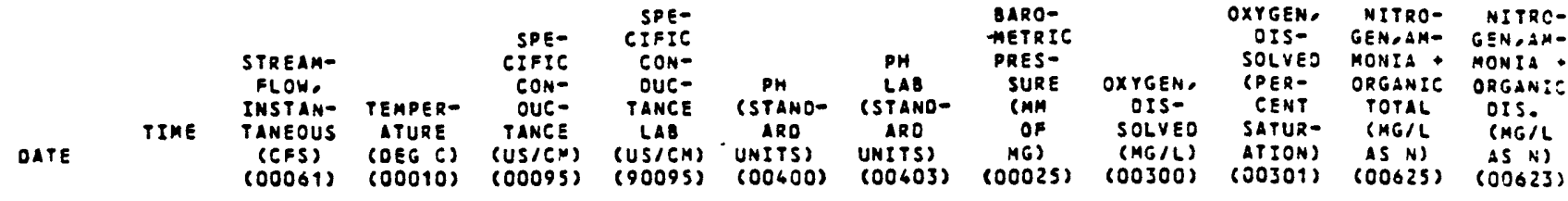

$\begin{array}{ll}\text { AUG } & 1986 \\ 21 \ldots \ldots & 1100 \\ 21 \ldots \ldots & 1200 \\ \text { SEP } & \\ 06 \ldots \ldots & 1630 \\ \text { APR } & 1985 \\ 06 \ldots \ldots & 1115 \\ 15 \ldots \ldots & 1645 \\ 15 \ldots \ldots & 1830 \\ 23 \ldots \ldots & 1600 \\ 29 \ldots \ldots & 1550 \\ M A Y & \\ 07 \ldots \ldots & 1365 \\ 15 \ldots \ldots & 1600 \\ 23 \ldots \ldots & 1630 \\ 28 \ldots \ldots & 1330 \\ \text { JUN } & \\ 06 \ldots . . & 1600 \\ \text { JUL } & \\ 10 \ldots \ldots & 1600\end{array}$

\begin{tabular}{|c|c|c|c|c|c|c|}
\hline $\begin{array}{l}1.9 \\
2.0\end{array}$ & 8.5 & 135 & $\begin{array}{r}136 \\
-0\end{array}$ & 7.8 & 8.0 & 605 \\
\hline 1.6 & 9.5 & 125 & 128 & 7.8 & 8.0 & 600 \\
\hline $\begin{array}{l}3.6 \\
5.0 \\
6.2 \\
3.3 \\
3.0\end{array}$ & $\begin{array}{l}6.0 \\
7.5 \\
6.0 \\
7.0 \\
7.0\end{array}$ & $\begin{array}{l}140 \\
115 \\
108 \\
122 \\
120\end{array}$ & $\begin{array}{l}150 \\
123 \\
196 \\
127 \\
129\end{array}$ & $\begin{array}{l}7.8 \\
7.8 \\
8.1 \\
7.8\end{array}$ & $\begin{array}{l}8.3 \\
7.9 \\
8.1 \\
8.1 \\
8.2\end{array}$ & $\begin{array}{l}596 \\
595 \\
603 \\
602\end{array}$ \\
\hline $\begin{array}{l}2.6 \\
2.1 \\
2.9 \\
1.9\end{array}$ & $\begin{array}{r}7.5 \\
8.0 \\
11.0 \\
8.0\end{array}$ & $\begin{array}{l}125 \\
150 \\
128\end{array}$ & $\begin{array}{l}129 \\
132 \\
160 \\
135\end{array}$ & $\begin{array}{l}8.0 \\
8.1 \\
8.0 \\
7.7\end{array}$ & $\begin{array}{l}8.2 \\
8.2 \\
8.1 \\
7.8\end{array}$ & $\begin{array}{l}602 \\
600 \\
604 \\
598\end{array}$ \\
\hline 1.3 & 8.5 & 129 & 134 & 8.0 & 8.1 & 603 \\
\hline .93 & 10.0 & 110 & 118 & 0.0 & 8.3 & 603 \\
\hline
\end{tabular}

\begin{tabular}{|c|c|c|c|}
\hline 9.1 & 98 & $\begin{array}{r}<.20 \\
.20\end{array}$ & $<.20$ \\
\hline 8.9 & 99 & - & C.22 \\
\hline $\begin{array}{l}-.- \\
9.3 \\
9.8 \\
9.7 \\
9.8\end{array}$ & $\begin{array}{r}-5 \\
99 \\
101 \\
101 \\
102\end{array}$ & $\begin{array}{l}.50 \\
.50 \\
.50 \\
.60 \\
.50\end{array}$ & $\begin{array}{l}.35 \\
.32 \\
.50 \\
.85 \\
.35\end{array}$ \\
\hline $\begin{array}{l}9.2 \\
9.3 \\
8.6 \\
9.3\end{array}$ & $\begin{array}{r}97 \\
100 \\
99 \\
100\end{array}$ & $\begin{array}{l}.60 \\
.20 \\
.20 \\
.30\end{array}$ & $\begin{array}{r}.32 \\
<.25 \\
.95 \\
.25\end{array}$ \\
\hline 9.1 & 99 & $\therefore 0$ & $.3:$ \\
\hline 8.5 & 95 & $<.20$ & <.2: \\
\hline
\end{tabular}

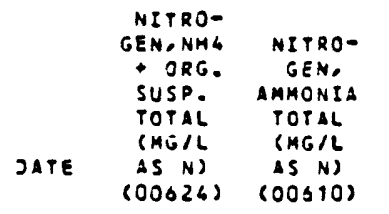

\begin{tabular}{|c|c|}
\hline $\begin{array}{l}\text { MITRO- } \\
\text { GEN } \\
\text { AMACNIA } \\
\text { OIS- } \\
\text { SOLVEO } \\
\text { SMGIL }\end{array}$ & $\begin{array}{l}\text { NITRO- } \\
\text { GEN. } \\
\text { ORGANIC } \\
\text { TOTAL } \\
\text { SHGIL }\end{array}$ \\
\hline $\begin{array}{c}S N) \\
(00008)\end{array}$ & $\begin{array}{c}15 \mathrm{~N}) \\
(00005)\end{array}$ \\
\hline
\end{tabular}

$\begin{array}{lc}\text { NITRO- } & \\ \text { GENE } & \text { NITRO- } \\ \text { ORGANIC } & \text { GEND } \\ \text { OIS- } & \text { NOZ }+ \text { NOS } \\ \text { SOLVEO } & \text { TOTAL } \\ \text { (MG/L } & \text { (MGIL } \\ \text { AS N) } & \text { AS N) }\end{array}$
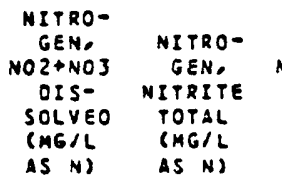

NITRO-

GEN, NITRITE

OIS-

SOLVEO

(MGIL

$(00607)(00630)$ (00631) (00615)

$\begin{array}{cc}A S N) & A S N) \\ (00613) & (00620)\end{array}$

\begin{tabular}{|c|c|c|c|c|c|c|c|c|c|c|c|}
\hline $\begin{array}{l}21 \ldots \ldots \\
21 \leq 0\end{array}$ & $\because$ & $\begin{array}{l}.009 \\
.006\end{array}$ & .005 & .0 & $\because$ & $\begin{array}{l}.04 \\
.05\end{array}$ & .04 & $\begin{array}{l}.005 \\
.003\end{array}$ & .007 & $\begin{array}{l}.04 \\
.05\end{array}$ & $\because 63$ \\
\hline ODR : & - & .006 & .006 & $-\cdot$ & -- & .06 & $<.01$ & .006 & .005 & .06 & -- \\
\hline $\begin{array}{l}06 \ldots . . \\
15 . \ldots \\
15 . \ldots \\
23 \ldots \\
29 . \ldots \\
\text { may }\end{array}$ & $\begin{array}{l}.30 \\
.30 \\
.10 \\
.120\end{array}$ & $\begin{array}{l}.024 \\
.038 \\
.043 \\
.039 \\
.032\end{array}$ & $\begin{array}{l}.022 \\
.037 \\
.032 \\
.034 \\
.043\end{array}$ & $\begin{array}{l}.58 \\
.56 \\
.56 \\
.36 \\
.47\end{array}$ & $\begin{array}{l}.28 \\
.26 \\
.67 \\
.20 \\
.26\end{array}$ & $\begin{array}{l}.09 \\
.11 \\
.12 \\
.07 \\
.00\end{array}$ & $\begin{array}{l}.09 \\
.11 \\
.12 \\
.07 \\
.06\end{array}$ & $\begin{array}{l}.002 \\
.002 \\
.006 \\
.006 \\
.003\end{array}$ & $\begin{array}{l}.003 \\
.004 \\
.004 \\
.002 \\
.003\end{array}$ & $\begin{array}{l}.01 \\
.10 \\
.12 \\
.37 \\
.00\end{array}$ & $\begin{array}{l}.02 \\
.11 \\
.12 \\
.67 \\
.65\end{array}$ \\
\hline $\begin{array}{c}07 \ldots . . \\
15 \ldots . \\
233 \ldots \\
23 \ldots \\
\text { JUN }\end{array}$ & $\begin{array}{r}10 \\
110 \\
.10\end{array}$ & $\begin{array}{l}.032 \\
.039 \\
.047 \\
.064\end{array}$ & $\begin{array}{r}.036 \\
.046 \\
.039\end{array}$ & $\begin{array}{l}.37 \\
.16 \\
.15 \\
.26\end{array}$ & $\begin{array}{l}.26 \\
=.05 \\
.16\end{array}$ & $\begin{array}{l}.06 \\
.02 \\
.02 \\
.03\end{array}$ & $\begin{array}{l}.04 \\
.04 \\
.03 \\
.03\end{array}$ & $\begin{array}{l}.002 \\
.009 \\
.003 \\
.001\end{array}$ & $\begin{array}{r}.001 \\
-0 \\
.004 \\
<.001\end{array}$ & $\begin{array}{l}.04 \\
.02 \\
.02 \\
.03\end{array}$ & $\begin{array}{l}.02 \\
0.02 \\
-0\end{array}$ \\
\hline JuL. & .10 & .050 & .052 & .35 & .25 & .03 & .03 & .003 & .008 & .03 & .02 \\
\hline 16. & -- & .031 & .034 & -- & -- & .07 & .07 & .008 & .010 & .09 & .03 \\
\hline
\end{tabular}

AUG, 1984

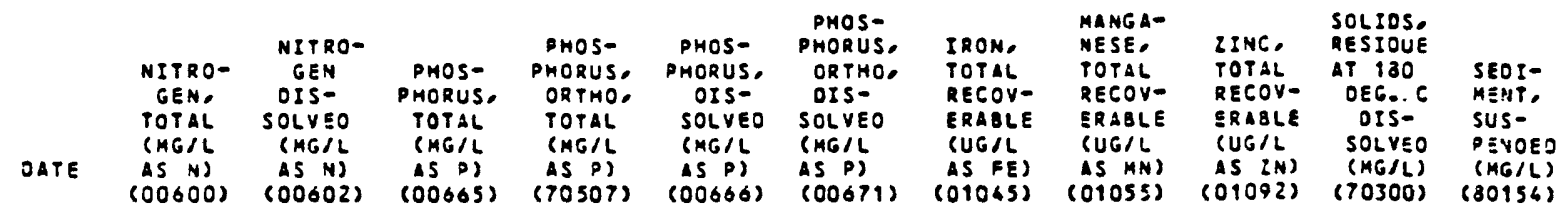

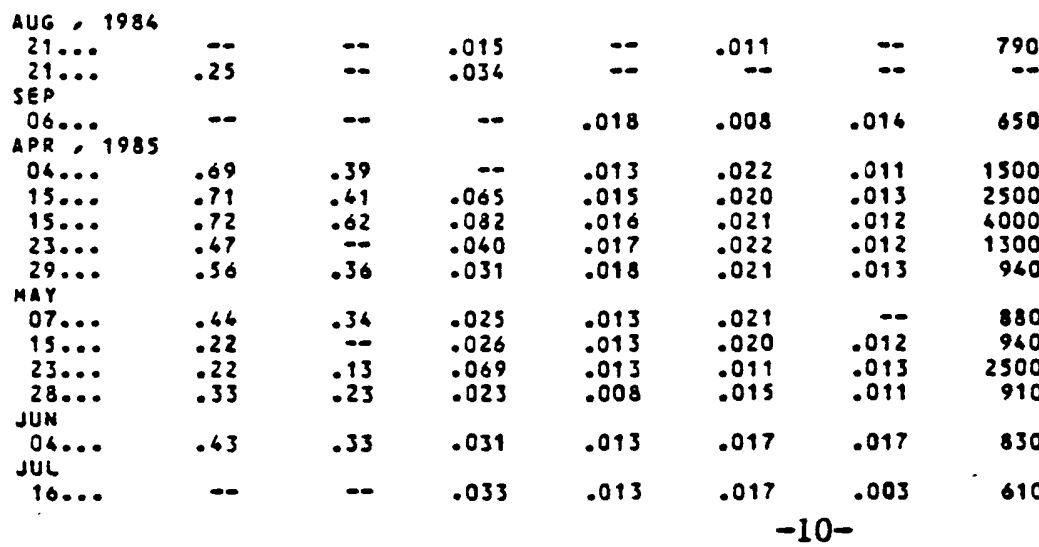


TABLE 1.-Water-quality and water-quantity data, August 1984-July 1985a-Continued

SITE 2 (EDGEWOOD CREEK BELOW HIGHWAY 50 NEAR STATELINE, NV; STA. 10336761)

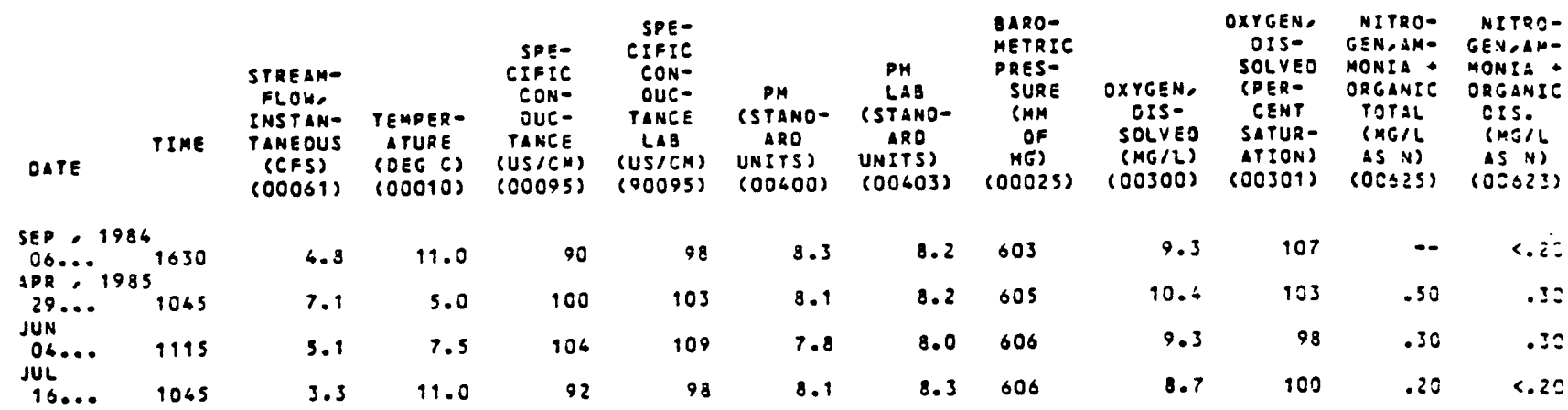

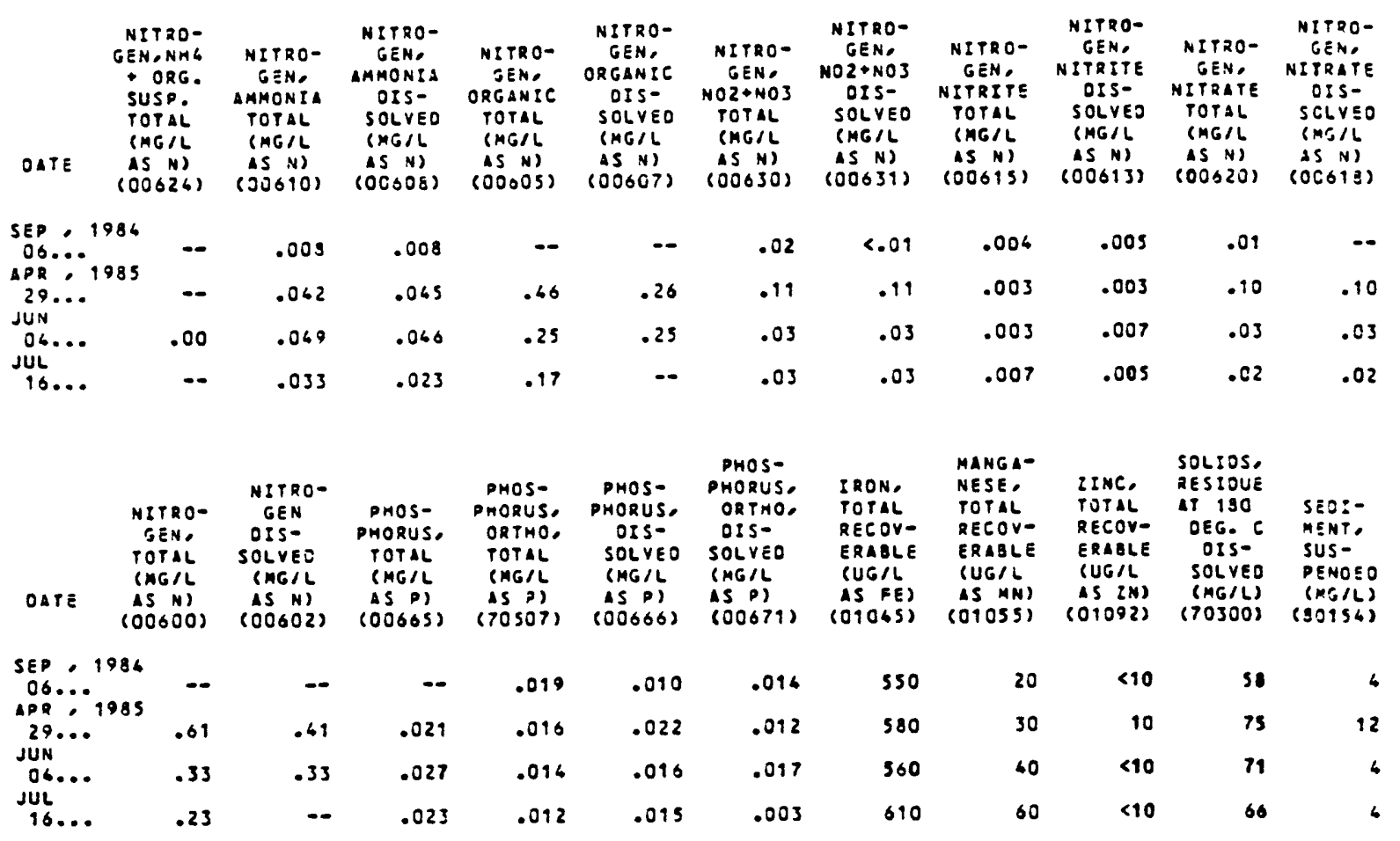


SITE 3 (EDGEWOOD CREEK AT LAKE TAHOE NEAR STATELINE, NV; STA. 10336765)

\begin{tabular}{|c|c|c|c|c|c|c|c|c|c|c|c|c|}
\hline DATE & TIME & $\begin{array}{l}\text { STREAM- } \\
\text { FLOHE } \\
\text { INSTAN- } \\
\text { TANEOUS } \\
\text { (CFS) } \\
\text { (OOOGI) }\end{array}$ & $\begin{array}{l}\text { TEMPER- } \\
\text { ATURE } \\
\text { (OEG C) } \\
(00010)\end{array}$ & $\begin{array}{l}\text { SPE- } \\
\text { CIFIC } \\
\text { CON- } \\
\text { OUC- } \\
\text { TANCE } \\
\text { (US/CN) } \\
\text { (COOSS) }\end{array}$ & $\begin{array}{l}\text { SPE- } \\
\text { CIFIC } \\
\text { CON- } \\
\text { OUC- } \\
\text { TANCE } \\
\text { LAB } \\
\text { (US/CA) } \\
\text { ( } 90095)\end{array}$ & $\begin{array}{l}\text { PH } \\
\text { (STANO- } \\
\text { ARO } \\
\text { UNITSS } \\
\text { (OOGOO) }\end{array}$ & $\begin{array}{c}P H \\
\text { LAB } \\
\text { (STANO- } \\
\text { ARO } \\
\text { UNITSS } \\
\text { (OO4O3) }\end{array}$ & $\begin{array}{l}\text { BARO- } \\
\text { METRIC } \\
\text { PRES- } \\
\text { SURE } \\
\text { (MM } \\
\text { OF } \\
\text { HG) } \\
(00025)\end{array}$ & $\begin{array}{c}\text { OXYGEN, } \\
\text { OIS- } \\
\text { SOLVEO } \\
\text { (MGIL) } \\
(00300)\end{array}$ & $\begin{array}{l}\text { OXYGEN- } \\
\text { OISS- } \\
\text { SOLVED } \\
\text { (PER- } \\
\text { CENT } \\
\text { SATUR- } \\
\text { ATION) } \\
\text { (OOJOIS }\end{array}$ & $\begin{array}{l}\text { NITRO- } \\
\text { GENIAM- } \\
\text { MONIA - } \\
\text { ORGANIC } \\
\text { TOTAL } \\
\text { (MGIL } \\
\text { AS H) } \\
\text { (OOO25) }\end{array}$ & $\begin{array}{l}\text { NITRO- } \\
\text { GENOAM- } \\
\text { MONIA } \\
\text { ORGANIC } \\
\text { OIS. } \\
\text { (UGIL } \\
\text { AS N) } \\
\text { (OOSZZ) }\end{array}$ \\
\hline $\begin{array}{l}\text { SEP. } \\
06 \ldots . . \\
\text { IDR }\end{array}$ & $5^{4} 1800$ & 3.8 & 20.0 & 100 & 106 & 9.3 & 9.1 & 605 & 10.1 & 161 & $\cdots$ & $<.20$ \\
\hline $\begin{array}{r}04 \ldots \\
95 \ldots \\
23 \ldots \ldots \\
29 \ldots \\
\text { YAY }\end{array}$ & $\begin{array}{l}1265 \\
1115 \\
1065 \\
1365\end{array}$ & $\begin{array}{l}12 \\
11 \\
8.1 \\
5.8\end{array}$ & $\begin{array}{r}7.0 \\
9.0 \\
9.0 \\
10.5\end{array}$ & $\begin{array}{l}127 \\
105 \\
107 \\
112\end{array}$ & $\begin{array}{l}135 \\
112 \\
112 \\
118\end{array}$ & $\begin{array}{l}-.7 \\
? .7 \\
8.0 \\
8.1\end{array}$ & $\begin{array}{l}8.0 \\
8.0 \\
8.2 \\
8.3\end{array}$ & $\begin{array}{l}605 \\
602 \\
606 \\
606\end{array}$ & $\begin{array}{r}10.2 \\
9.7 \\
10.8 \\
10.6\end{array}$ & $\begin{array}{l}106 \\
107 \\
118 \\
120\end{array}$ & $\begin{array}{l}.90 \\
1.0 \\
.50 \\
.90\end{array}$ & $\begin{array}{l}.22 \\
.40 \\
.60 \\
.30\end{array}$ \\
\hline $\begin{array}{l}07 \ldots \\
15 \ldots \\
23 \ldots \\
28 \ldots \\
\text { JUN }\end{array}$ & $\begin{array}{l}1030 \\
1100 \\
1130 \\
1015\end{array}$ & $\begin{array}{l}4.0 \\
6.8 \\
6.5 \\
5.6\end{array}$ & $\begin{array}{l}11.5 \\
11.0 \\
16.5 \\
12.0\end{array}$ & $\begin{array}{l}-\infty \\
110 \\
165 \\
125\end{array}$ & $\begin{array}{l}115 \\
116 \\
134 \\
117\end{array}$ & $\begin{array}{l}8.4 \\
8.4 \\
8.7 \\
8.7\end{array}$ & $\begin{array}{l}8.5 \\
8.5 \\
-.5 \\
8.6\end{array}$ & $\begin{array}{l}606 \\
605 \\
606 \\
603\end{array}$ & $\begin{array}{r}9.6 \\
10.0 \\
9.1 \\
9.0\end{array}$ & $\begin{array}{l}111 \\
115 \\
113 \\
106\end{array}$ & $\begin{array}{l}.30 \\
.30 \\
.60 \\
.30\end{array}$ & $\begin{array}{l}.20 \\
.30 \\
.60 \\
.30\end{array}$ \\
\hline $\begin{array}{l}06 \ldots . . . \\
\text { JUL } \\
16 \ldots . . .\end{array}$ & $\begin{array}{l}0845 \\
0915\end{array}$ & $\begin{array}{l}4.6 \\
1.2\end{array}$ & $\begin{array}{l}11.0 \\
18.0\end{array}$ & $\begin{array}{l}110 \\
108\end{array}$ & 116 & $\begin{array}{l}8.7 \\
9.3\end{array}$ & $\begin{array}{l}8.6 \\
9.3\end{array}$ & $\begin{array}{l}605 \\
606\end{array}$ & $\begin{array}{l}9.1 \\
8.0\end{array}$ & 104 & $\begin{array}{r}.30 \\
.30\end{array}$ & $\begin{array}{l}.30 \\
.32\end{array}$ \\
\hline
\end{tabular}

\begin{tabular}{|c|c|c|c|c|c|c|c|c|c|c|c|}
\hline & $\begin{array}{l}\text { NITRO- } \\
\text { GENONMG } \\
\text { + ORG. } \\
\text { SUSP. } \\
\text { POTAL } \\
\text { CMGIL }\end{array}$ & $\begin{array}{l}\text { NITRO- } \\
\text { GEND } \\
\text { AMHONIA } \\
\text { TOTAL } \\
\text { GHG/L }\end{array}$ & $\begin{array}{l}\text { NITRO- } \\
\text { GEN } \\
\text { AMMONIA } \\
\text { OIS- } \\
\text { SOLVED } \\
\text { SMGIL }\end{array}$ & $\begin{array}{l}\text { MITRO- } \\
\text { GEN. } \\
\text { ORGANIC } \\
\text { TOTAL } \\
\text { PMG/L }\end{array}$ & $\begin{array}{l}\text { NITRO- } \\
\text { GEND } \\
\text { ORGANIC } \\
\text { OIS- } \\
\text { SOLVEO } \\
\text { GMGIL }\end{array}$ & $\begin{array}{l}\text { NITRO- } \\
\text { GEND } \\
\text { NOZ }+ \text { NOS } \\
\text { TOTAL } \\
\text { CMG/L }\end{array}$ & $\begin{array}{c}\text { NITRO- } \\
\text { GENO } \\
\text { NOL+NOS } \\
\text { OIS- } \\
\text { SOLVEO } \\
\text { SHEIL }\end{array}$ & $\begin{array}{l}\text { NITRO- } \\
\text { GEN } \\
\text { NITRITE } \\
\text { TOTAL } \\
\text { CHEIL }\end{array}$ & $\begin{array}{l}\text { NITRO- } \\
\text { GENE } \\
\text { NITRITE } \\
\text { OIS- } \\
\text { SOLVEO } \\
\text { CMGIL }\end{array}$ & $\begin{array}{l}\text { NITRO- } \\
\text { GEN, } \\
\text { NITRATE } \\
\text { TOTAL } \\
\text { ONG/L }\end{array}$ & $\begin{array}{l}\text { NITRO- } \\
\text { SEND } \\
\text { MITRATE } \\
\text { DISO } \\
\text { SOLVEO } \\
\text { CMGIL }\end{array}$ \\
\hline & $\begin{array}{c}15 N) \\
(00026)\end{array}$ & $\begin{array}{c}\text { (S N) } \\
(00610)\end{array}$ & $\begin{array}{l}\text { (S N) } \\
(00608)\end{array}$ & $\begin{array}{c}\text { is Ni) } \\
(00005)\end{array}$ & $\begin{array}{c}15 N) \\
(00007)\end{array}$ & $\begin{array}{c}15 N) \\
(00630)\end{array}$ & $\begin{array}{c}15 N) \\
(00631)\end{array}$ & $\begin{array}{c}15 N) \\
(00015)\end{array}$ & $\begin{array}{c}\operatorname{CSN}) \\
(00613)\end{array}$ & $\begin{array}{c}15 \mathrm{~N}) \\
(00020)\end{array}$ & $\begin{array}{c}25 N) \\
(00018)\end{array}$ \\
\hline
\end{tabular}

SEP. 1984

OPR: 1985

Q $1985+2$

$15 . \cdots, \quad .60$

$23 \ldots . .10$

$.05:$

.047

00608

(00005)

$(00007)$

$(00630)$

$(00631)$

(00015)

(00013)

$(00020)$

(OCo18)

MAY

0?... . 10

$15 \ldots . .00$

$23 \ldots .00$

$28 \ldots$

06.

06... . .00

$16 . .$.

.088
.055
.056
.056
.047
.037
.041
.038
.047
.046
.031

.091
.048
.046
.046
.041
.030
.026
.046
.043
.023

.0
.85
.95
.45
.85
.26
.26
.36
.25
.25
.27

$\begin{array}{ll}.0 & <.01 \\ .15 & .15 \\ .35 & .16 \\ .35 & .10 \\ .26 & .08 \\ .17 & .03 \\ .0 & .03 \\ .38 & .02 \\ .25 & .01 \\ .26 & .02 \\ .28 & <.01\end{array}$

$<.01$
.16
.14
.09
.08
.03
.03
.01
.01
.02
.01

.005

.007

.005

.005

$<.001$

.002

.004

.006

.006

.005

.002

.008
.002

.002
.003

.003

.006

.008

.006

.006

$\begin{array}{ll}.16 & .16 \\ .16 & .09 \\ .09 & .09 \\ .07 & .07\end{array}$

$.02 \quad .03$

$.03 \quad .02$

$.00 \quad .01$

$.02 \quad .31$

-. .00

\begin{tabular}{|c|c|c|c|c|c|c|c|c|c|c|c|}
\hline DATE & $\begin{array}{l}\text { NITRO- } \\
\text { GENO } \\
\text { TOTAL } \\
\text { (NEAL } \\
\text { AS N) } \\
\text { (OOOOOO) }\end{array}$ & $\begin{array}{l}\text { NITRO- } \\
\text { GEN } \\
\text { OIS- } \\
\text { SOLVEO } \\
\text { (NG/L } \\
\text { AS N) } \\
(00002)\end{array}$ & $\begin{array}{l}\text { PMOS- } \\
\text { PHORUS } \\
\text { TOTAL } \\
\text { (MG/L } \\
\text { AS D) } \\
\text { (00665) }\end{array}$ & $\begin{array}{l}\text { PHOS- } \\
\text { PMORUSO } \\
\text { ORTHO. } \\
\text { TOTAL } \\
\text { (NGIL } \\
\text { AS P) } \\
\text { (TOSOT) }\end{array}$ & $\begin{array}{l}\text { PHOS- } \\
\text { PHORUS. } \\
\text { OIS- } \\
\text { SOLVEO } \\
\text { (NG/L } \\
\text { AS P) } \\
(00606)\end{array}$ & $\begin{array}{l}\text { PHOS- } \\
\text { PMORUS, } \\
\text { ORTHO. } \\
\text { OIS- } \\
\text { SOLVEO } \\
\text { (MG/L } \\
\text { AS P) } \\
\text { (OO6PI) }\end{array}$ & $\begin{array}{l}\text { IRON } \\
\text { TOTAL } \\
\text { RECOV- } \\
\text { ERABLE } \\
\text { (UG/L } \\
\text { AS FE) } \\
\text { (O1OLS) }\end{array}$ & $\begin{array}{l}\text { MANGA- } \\
\text { NESE, } \\
\text { POTAL } \\
\text { RECOY- } \\
\text { ERABLE } \\
\text { (UG/L } \\
\text { AS MNS } \\
\text { (OIOSS) }\end{array}$ & $\begin{array}{l}\text { ZINCE } \\
\text { TOTAL } \\
\text { RECOV- } \\
\text { ERABLE } \\
\text { (UG IL } \\
\text { AS ZN) } \\
\text { (OIOSZ) }\end{array}$ & $\begin{array}{l}\text { SOLIJS, } \\
\text { RESIOUE } \\
\text { AT } 180 \\
\text { OES.C } \\
\text { OIS- } \\
\text { SOLVEO } \\
\text { (MG/L) } \\
\text { (7OJOO) }\end{array}$ & $\begin{array}{l}\text { SEJI- } \\
\text { MENT, } \\
\text { SUS- } \\
\text { PENOEE } \\
\text { (MEIL) } \\
\text { (BOTSL) }\end{array}$ \\
\hline $\begin{array}{l}\text { SEP } . \\
\text { OG... } \\
\text { APR }\end{array}$ & $\begin{array}{l}1986 \\
1985\end{array}$ & -- & - & .082 & -- & .073 & 460 & 20 & $<10$ & 68 & 6 \\
\hline $\begin{array}{l}06 \ldots \\
15 \ldots \ldots \\
23 \ldots \ldots \\
29 \ldots \\
\text { mar }\end{array}$ & $\begin{array}{l}1.0 \\
1.1 \\
.60 \\
.98\end{array}$ & $\begin{array}{l}.34 \\
.54 \\
.49 \\
.38\end{array}$ & $\begin{array}{l}.066 \\
.030 \\
.035 \\
.031\end{array}$ & $\begin{array}{l}.027 \\
.017 \\
.020 \\
.023\end{array}$ & $\begin{array}{l}.032 \\
.022 \\
.027 \\
.027\end{array}$ & $\begin{array}{l}.021 \\
.016 \\
.015 \\
.017\end{array}$ & $\begin{array}{r}1600 \\
820 \\
670 \\
480\end{array}$ & $\begin{array}{r}70 \\
50 \\
50 \\
180\end{array}$ & $\begin{array}{l}30 \\
30 \\
10 \\
80\end{array}$ & $\begin{array}{l}89 \\
77 \\
79 \\
85\end{array}$ & $\begin{array}{r}20 \\
8 \\
6 \\
6\end{array}$ \\
\hline $\begin{array}{l}07 \ldots \\
15 \ldots \\
23 \ldots \\
28 \ldots \\
\text { JUN }\end{array}$ & $\begin{array}{l}.33 \\
.33 \\
.62 \\
.31\end{array}$ & $\begin{array}{l}.23 \\
.33 \\
.61 \\
.31\end{array}$ & $\begin{array}{l}.031 \\
.032 \\
.029 \\
.026\end{array}$ & $\begin{array}{l}.015 \\
.015 \\
.008 \\
.009\end{array}$ & $\begin{array}{l}.022 \\
.026 \\
.015 \\
.013\end{array}$ & $\begin{array}{l}.011 \\
.012 \\
.005 \\
.010\end{array}$ & $\begin{array}{l}680 \\
690 \\
680 \\
520\end{array}$ & $\begin{array}{l}50 \\
60 \\
60 \\
60\end{array}$ & $\begin{array}{r}40 \\
10 \\
10 \\
<10\end{array}$ & $\begin{array}{l}82 \\
86 \\
90 \\
80\end{array}$ & $\begin{array}{l}6 \\
6 \\
3\end{array}$ \\
\hline JUL & .32 & .32 & .031 & .094 & .017 & .013 & 560 & 40 & $<10$ & 77 & 6 \\
\hline - & - & .31 & .061 & .026 & .032 & .004 & 580 & 60 & 20 & 76 & 6 \\
\hline
\end{tabular}




\begin{tabular}{|c|c|c|c|c|c|c|c|c|c|c|c|c|}
\hline & IME & $\begin{array}{l}\text { STREAM- } \\
\text { FLOHE } \\
\text { INSTAN- } \\
\text { TANEOUS }\end{array}$ & $\begin{array}{l}\text { TEAPER- } \\
\text { ATURE }\end{array}$ & $\begin{array}{l}\text { SDE- } \\
\text { CIEIC } \\
\text { CON- } \\
\text { OUC- } \\
\text { TANCE }\end{array}$ & $\begin{array}{l}\text { SPE- } \\
\text { CIEIC } \\
\text { CON- } \\
\text { OUC- } \\
\text { TANCE } \\
\text { LAB }\end{array}$ & $\begin{array}{c}\text { PH } \\
\text { CSTANO- } \\
\text { ARO }\end{array}$ & $\begin{array}{l}\text { PH } \\
\text { LAB } \\
\text { CSTANO- } \\
\text { ARO }\end{array}$ & $\begin{array}{l}\text { BARO- } \\
\text { METRIC } \\
\text { PRES- } \\
\text { SURE } \\
\text { IMM } \\
\text { OF }\end{array}$ & $\begin{array}{l}\text { OXYGEN, } \\
\text { OIS- } \\
\text { SOLVEO }\end{array}$ & $\begin{array}{l}\text { OXYGEN } \\
\text { DIS- } \\
\text { SOLVEC } \\
\text { SPER- } \\
\text { CENT } \\
\text { SATUR- }\end{array}$ & $\begin{array}{l}\text { NITRO- } \\
\text { GENEAM- } \\
\text { MONIA } \\
\text { ORGANSC } \\
\text { TOTAL } \\
\text { SNGIL }\end{array}$ & $\begin{array}{l}\text { NITRO- } \\
\text { GEN,AM- } \\
\text { MON :A } \\
\text { ORGANIE } \\
\text { OISE } \\
\text { CMGIL }\end{array}$ \\
\hline e & & $\begin{array}{c}(C F 5) \\
(00061)\end{array}$ & $\begin{array}{l}\text { (DEC C) } \\
(00010)\end{array}$ & $\begin{array}{l}(U S / C N) \\
(00095)\end{array}$ & $\begin{array}{l}\text { (USICH) } \\
(90095)\end{array}$ & $\begin{array}{l}\text { UNITS) } \\
\text { (00600) }\end{array}$ & $\begin{array}{l}\text { UNITS) } \\
(00603)\end{array}$ & $\begin{array}{c}H G) \\
(00025)\end{array}$ & $\begin{array}{r}(M G / L) \\
(00300)\end{array}$ & $\begin{array}{l}\text { ATION) } \\
\text { (00301) }\end{array}$ & $\begin{array}{c}\text { A5 N) } \\
(00625)\end{array}$ & $\begin{array}{c}45 N) \\
(00623)\end{array}$ \\
\hline
\end{tabular}

\begin{tabular}{|c|c|c|c|c|}
\hline $\begin{array}{l}30 \\
A P R\end{array}$ & 1515 & $-\infty$ & $-\infty$ & 192 \\
\hline $\begin{array}{l}06 \ldots . . \\
06 \ldots \\
13 \ldots \\
23 \ldots \\
29 \ldots\end{array}$ & $\begin{array}{l}0830 \\
1115 \\
0930 \\
0830 \\
0830\end{array}$ & $\begin{array}{l}=- \\
=- \\
=- \\
--\end{array}$ & $\begin{array}{r}10.0 \\
16.5 \\
10.5 \\
12.0\end{array}$ & $\begin{array}{l}150 \\
154 \\
185 \\
262 \\
308\end{array}$ \\
\hline $\begin{array}{c}\text { MaY } \\
07 \ldots . . .\end{array}$ & 0900 & $-\infty$ & 15.0 & 335 \\
\hline $\begin{array}{l}\text { JUN } \\
\text { O6. } \\
\text { JUL }\end{array}$ & 1545 & -- & 23.0 & 683 \\
\hline $\begin{array}{l}26 \ldots \\
26 \ldots\end{array}$ & $\begin{array}{l}1660 \\
1650\end{array}$ & $\begin{array}{l}\text { E. } 75 \\
\text { E. } 25\end{array}$ & 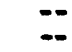 & $\begin{array}{l}164 \\
158\end{array}$ \\
\hline
\end{tabular}

\begin{tabular}{|c|c|c|c|c|c|c|c|c|}
\hline 208 & $-\infty$ & 7.5 & & - & $-\infty$ & $-\infty$ & $.3 C$ & .20 \\
\hline $\begin{array}{l}164 \\
162 \\
196 \\
269 \\
321\end{array}$ & $\begin{array}{r}-0 \\
-0 \\
9.2 \\
8.0 \\
8.0\end{array}$ & $\begin{array}{l}7.8 \\
8.7 \\
8.8 \\
7.7 \\
7.8\end{array}$ & $\begin{array}{l}600 \\
598 \\
602 \\
603\end{array}$ & $-\infty$ & $\begin{array}{r}11.6 \\
-.5 \\
9.9 \\
8.8 \\
9.5\end{array}$ & $\begin{array}{r}129 \\
126 \\
126 \\
112\end{array}$ & $\begin{array}{l}1.2 \\
1.0 \\
.50 \\
.90 \\
.70\end{array}$ & $\begin{array}{l}.50 \\
.30 \\
.32 \\
.70 \\
.30\end{array}$ \\
\hline 362 & 8.2 & 8.1 & 603 & & 8.7 & 110 & - & .42 \\
\hline 486 & 7.9 & 7.7 & 606 & & 7.3 & 105 & 1.0 & .50 \\
\hline- & $-\infty$ & $-\infty$ & & & - & - & $=$ & \\
\hline-- & $\infty$ & $-\infty$ & & $-\infty$ & $-\infty$ & 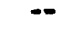 & 6.5 & \\
\hline
\end{tabular}

\begin{tabular}{|c|c|c|}
\hline & $\begin{array}{l}\text { NITRO- } \\
\text { GEN,NHG } \\
\text { SORG. } \\
\text { SUSP. } \\
\text { TOTAL } \\
\text { SNGIL }\end{array}$ & $\begin{array}{l}\text { NITRO- } \\
\text { GEN } \\
\text { AMMONIA } \\
\text { TOTAL } \\
\text { SMGIL }\end{array}$ \\
\hline ITE & $\begin{array}{c}A S N) \\
(00626)\end{array}$ & $\begin{array}{c}A 5 N) \\
(00610)\end{array}$ \\
\hline
\end{tabular}

NITROGEN, MMONI: OISSOLVEO (MG/L a $N)$ (0060s)

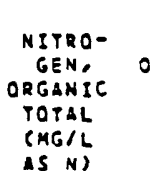

NITROGEN, NITROORGANIC ORGANIC
OISSOLVEO (0060s) ( $M G / L$

$\begin{array}{ll}\text { (MG/L } & \text { (MGIL } \\ \text { ASN N } & \text { AS N) }\end{array}$

(00607) (00630)
NITROGEN. NO2 + NO3 OISSOLVED CMG/L AS N) (OC631) (00615)
NITROGEN. nITRITE TOTAL (HG/L
NIPRO-

GENe GENE
NTRITE OISSOLVEO NITRATE SUESO TOTAL 4TERL (MG/L (00693) (00520)

NIT?OGEN, NTTOATE OISSCLVEO (MG/L (S N)

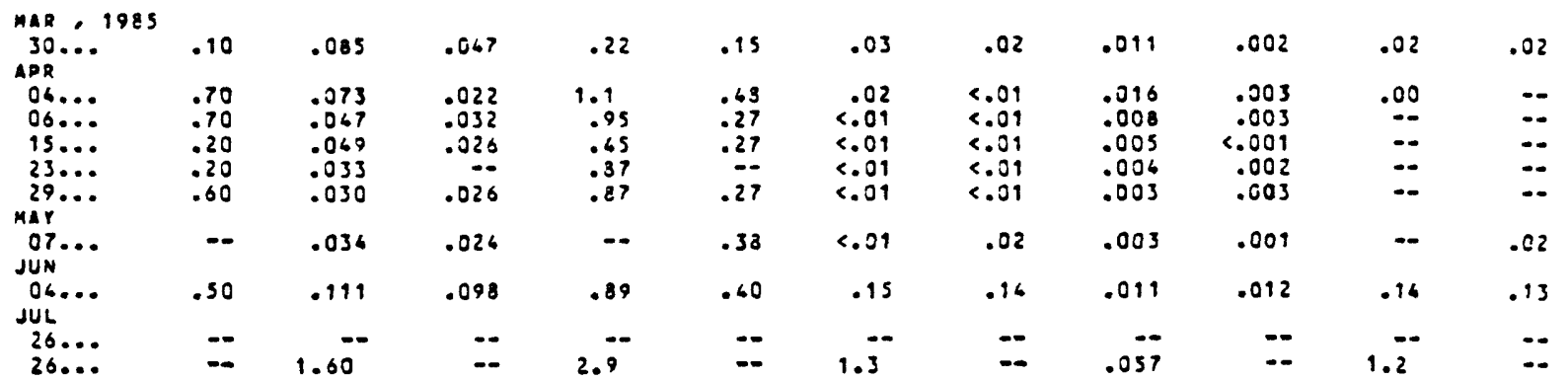

\begin{tabular}{|c|c|c|c|c|c|c|c|c|c|c|c|}
\hline DATE & $\begin{array}{l}\text { NITRO- } \\
\text { GENE } \\
\text { TOTAL } \\
\text { (AGNL } \\
\text { AS N) } \\
\text { (OOOOO) }\end{array}$ & $\begin{array}{l}\text { NITRO- } \\
\text { GEN } \\
\text { OIS- } \\
\text { SOLVEO } \\
\text { (MGIL } \\
\text { AS N) } \\
\text { (OOSOL }\end{array}$ & $\begin{array}{l}\text { PHOS- } \\
\text { PHORUSE } \\
\text { TOTAL } \\
\text { (AG/L } \\
\text { AS P) } \\
\text { (00665) }\end{array}$ & $\begin{array}{l}\text { PHOS- } \\
\text { PHORUSE } \\
\text { ORTHO. } \\
\text { TOTAL } \\
\text { (MGIL } \\
\text { AS D) } \\
\text { (7OSOT) }\end{array}$ & $\begin{array}{l}\text { PHOS- } \\
\text { PHORUSE } \\
\text { OIS- } \\
\text { SOLVEO } \\
\text { (MGIL } \\
\text { AS P) } \\
\text { (OOG66) }\end{array}$ & $\begin{array}{l}\text { PHOS- } \\
\text { PHORUS. } \\
\text { ORTHO. } \\
\text { OIS- } \\
\text { SOLVEO } \\
(\text { MG/L } \\
\text { AS P) } \\
(00671)\end{array}$ & $\begin{array}{l}\text { IRONO } \\
\text { TOYAL } \\
\text { RECOY- } \\
\text { ERABLE } \\
\text { (UG/L } \\
\text { AS FE) } \\
\text { (O1OGS) }\end{array}$ & $\begin{array}{l}\text { MANGA- } \\
\text { NESE. } \\
\text { TOTAL } \\
\text { RECOV- } \\
\text { ERABLE } \\
\text { (UGIL } \\
\text { IS MN) } \\
\text { (OSOSS) }\end{array}$ & $\begin{array}{l}\text { ZINCE } \\
\text { TOTAL } \\
\text { RECOV- } \\
\text { ERABLE } \\
\text { (UG/L } \\
\text { AS ZN) } \\
\text { (O1O9Z) }\end{array}$ & $\begin{array}{l}\text { SOL IOS, } \\
\text { RESIDUE } \\
\text { AT } 180 \\
\text { DEG. C } \\
\text { OIS- } \\
\text { SOLVEO } \\
\text { (MGIL) } \\
\text { (7OSOO) }\end{array}$ & $\begin{array}{l}\text { SEOI- } \\
\text { MENT. } \\
\text { SUS- } \\
\text { DENOEO } \\
\text { (MG/L) } \\
\text { (3OISG) }\end{array}$ \\
\hline \multicolumn{12}{|c|}{ MAR , 1985} \\
\hline $\begin{array}{l}30 \\
A P R\end{array}$ & .33 & .22 & .039 & .013 & .005 & .003 & 1200 & 130 & 30 & 131 & - \\
\hline $\begin{array}{l}06 \ldots \\
06 \ldots \\
15 \ldots \ldots \\
23 \ldots \ldots \\
29 \ldots \ldots \\
\text { MAY }\end{array}$ & $\begin{array}{r}1.2 \\
=- \\
-- \\
- \\
-\end{array}$ & $\begin{array}{l}-\infty \\
-- \\
-\infty \\
-\infty\end{array}$ & $\begin{array}{l}.161 \\
.110 \\
.035 \\
.075 \\
.046\end{array}$ & $\begin{array}{l}.028 \\
.013 \\
.008 \\
.022 \\
.016\end{array}$ & $\begin{array}{l}.013 \\
.012 \\
.013 \\
.016 \\
.016\end{array}$ & $\begin{array}{l}.005 \\
.002 \\
.003 \\
.002 \\
.004\end{array}$ & $\begin{array}{r}5200 \\
3300 \\
600 \\
600 \\
390\end{array}$ & $\begin{array}{r}160 \\
90 \\
50 \\
150 \\
170\end{array}$ & $\begin{array}{l}80 \\
40 \\
20 \\
10 \\
20\end{array}$ & $\begin{array}{r}91 \\
99 \\
115 \\
161 \\
188\end{array}$ & $\begin{array}{r}37 \\
-0 \\
12 \\
8 \\
8\end{array}$ \\
\hline$\underset{\text { JUN }}{07 \cdots}$ & $\cdots$ & .62 & .031 & .008 & .011 & $<.001$ & 280 & $130^{\circ}$ & 20 & 229 & 6 \\
\hline $\begin{array}{l}\text { OL... } \\
\text { JUL }\end{array}$ & 1.2 & .66 & .083 & .025 & .013 & .008 & 1300 & 620 & 40 & 290 & 13 \\
\hline $\begin{array}{l}26 \ldots \\
26 \ldots\end{array}$ & 5.8 & 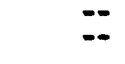 & .990 & .270 & $\ddot{-\infty}$ & $\ddot{-a}$ & $\begin{array}{r}26000 \\
-0\end{array}$ & 1600 & $\begin{array}{r}10 \\
-\infty\end{array}$ & 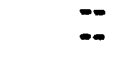 & $\begin{array}{l}202 \\
516\end{array}$ \\
\hline
\end{tabular}

a Hereln, the termg anmonia- and $2 \mathrm{AH}_{4}^{-}$both signify the combined concentration of annonta attrogen plus ammonium nitrogen (see text). Total and dissolved concentrations of ammonia" attrogen plus organic nitrogen, and of ammonia- nitrogen alone, were deternined analytically, and che concentrations of organie nitrogen there then calculated by dffference. The same procedure was used to calculate nitrate nitrogen on the basis of analytical daca for nitrite nitrogen plus nitrate nitrogen and for nitrite nitrogen alone. Values for total aitrogen (parameter code 00600) represent the calculated sua of the values for total anmia- udtrogen, total organic nitrogen, cocal nitrite nitrogen, and total nitrace atcrogen. Sinilarly, valuee for dissolved nitrogen (paramecer code 00602 ) represent the calculated sun of the four dissolved species. 
TABLE 2.-Seleoted oumary statistics for constituents and characteristics

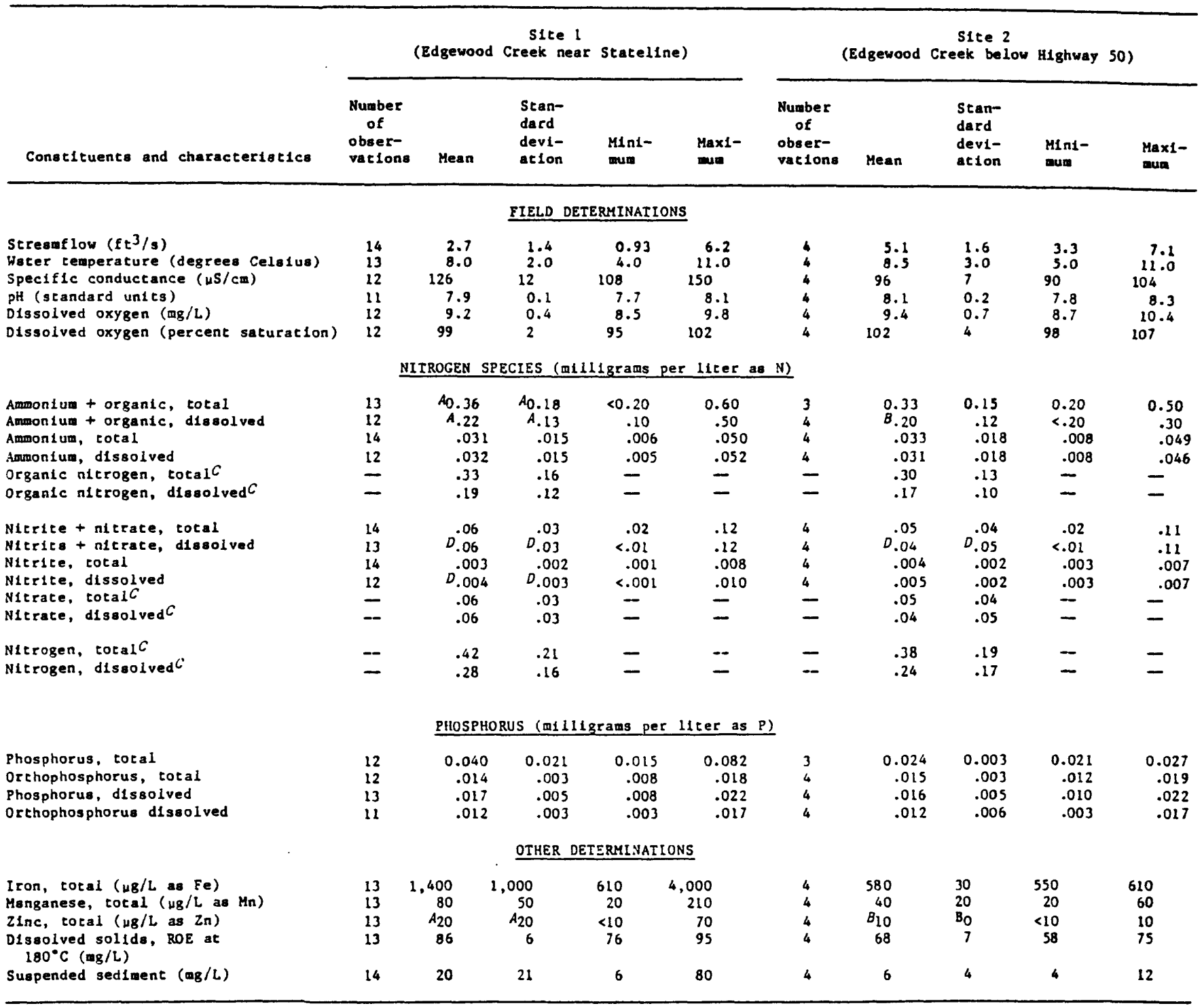

A Value estimated using the "log-probabilfty regression" method, as discussed by G11110n and Helsel (1984) and Helsel and Gil1100 (1985).

$B$ Concentrations below the detection lintt were assumed to be equal to one half of the detection ilmit for purposes of statistical calculations.

$C$ see footnoce $a$ In table 1. The means and standard deviations for total and dissolved organic nitrogen are the calculated differences between the statistical values for ammonium + organic nitrogen and for ammonium alone. Similarly, those for total and dissolved nitrate are calculated using the values for nitrite + nitrate and for nitrite alone. Finally, the means and standsrd devistions for total nitrogen or dissolved nitrogen are the sums of the values for ammonium + organic nitrogen plus those for nitrite + nitrate. 
TABLE 2.-Selected sumary statistics for constituents and characteristics-Continued

\begin{tabular}{|c|c|c|c|c|c|c|c|c|c|c|}
\hline \multirow[b]{2}{*}{ Constituents and characteristics } & \multicolumn{2}{|c|}{ (Edgewood } & \multicolumn{3}{|c|}{$\begin{array}{l}\text { site } 3 \\
\text { Creek at Lake Tahoe) }\end{array}$} & \multicolumn{2}{|c|}{ (Sediment-catchment } & \multicolumn{2}{|c|}{$\begin{aligned} \text { Site } 4 \\
\text { basin neer Tahoe }\end{aligned}$} & \multirow{2}{*}{$\begin{array}{c}{\text { V11lage })^{b}}^{\boldsymbol{M}^{\prime}} \\
\operatorname{Maxi}_{\operatorname{mum}}\end{array}$} \\
\hline & $\begin{array}{l}\text { Number } \\
\text { of } \\
\text { obser- } \\
\text { vationa }\end{array}$ & Mean & $\begin{array}{l}\text { Stan- } \\
\text { dard } \\
\text { devi- } \\
\text { ation }\end{array}$ & $\begin{array}{l}\text { Mini- } \\
\text { aum }\end{array}$ & $\begin{array}{l}\text { Maxi- } \\
\text { mum }\end{array}$ & $\begin{array}{l}\text { Number } \\
\text { of } \\
\text { obser- } \\
\text { vations }\end{array}$ & Mean' & $\begin{array}{l}\text { Stan- } \\
\text { dard } \\
\text { devi- } \\
\text { ation }\end{array}$ & $\begin{array}{l}\text { Mini- } \\
\text { mux }\end{array}$ & \\
\hline \multicolumn{11}{|c|}{ FIELD DETERMINATIONS } \\
\hline $\begin{array}{l}\left.\text { Streamflow ( } \mathrm{ft}^{3} / \mathrm{s}\right) \\
\text { Water temperature (degrees Celslus) } \\
\text { Speciflc conductance ( } \mathrm{S} / \mathrm{cm} \text { ) } \\
\text { pH (standard units) } \\
\text { Dissolved oxygen (mg/L) } \\
\text { Dissolved Oxygen } \\
\quad \text { (percent saturation) }\end{array}$ & $\begin{array}{l}11 \\
11 \\
10 \\
10 \\
11 \\
11\end{array}$ & $\begin{array}{r}5.9 \\
12.0 \\
115 \\
8.5 \\
9.6 \\
113^{-6}\end{array}$ & $\begin{array}{l}3.2 \\
4.0 \\
14^{.5} \\
.8\end{array}$ & $\begin{array}{r}1.2 \\
7.0 \\
100 \\
7.7 \\
8.0 \\
104\end{array}$ & $\begin{array}{r}12 \\
20.0 \\
145 \\
9.3 \\
10.8 \\
141\end{array}$ & $\begin{array}{l}- \\
6 \\
8 \\
5 \\
6 \\
6\end{array}$ & $\begin{array}{l}-74.0 \\
259 \\
F_{8.0} .0 \\
9.3 \\
114\end{array}$ & $\begin{array}{l}5.0 \\
114 \\
0.5 \\
11.4\end{array}$ & $\begin{array}{c}7 \overline{10.0} \\
150 \\
7.9 \\
7.3 \\
100\end{array}$ & $\begin{array}{r}\overline{23.0} \\
483 \\
9.2 \\
11.4 \\
129\end{array}$ \\
\hline \multicolumn{11}{|c|}{ NITROCEN SPECIES (m1111grams per Hiter as N) } \\
\hline $\begin{array}{l}\text { Ammonium + organic, total } \\
\text { Ammonium + organic, dissolved } \\
\text { Ammonium, cotal } \\
\text { Ammonium, dissolved } \\
\text { Organic nitrogen, totalC } \\
\text { Organic nitrogen, disaolvedC }\end{array}$ & $\begin{array}{l}10 \\
11 \\
11 \\
10 \\
-\end{array}$ & $\begin{array}{l}0.52 \\
A .30 \\
.049 \\
.044 \\
.47 \\
.26\end{array}$ & $\begin{array}{l}0.29 \\
. .10 \\
.015 \\
.019 \\
.28 \\
.08\end{array}$ & $\begin{array}{l}0.30 \\
<.20 \\
.031 \\
.023 \\
- \\
-\end{array}$ & $\begin{array}{l}1.0 \\
.40 \\
.088 \\
.091 \\
-- \\
-\end{array}$ & $\begin{array}{l}7 \\
8 \\
8 \\
7 \\
-- \\
-\end{array}$ & $\begin{array}{l}0.83 \\
.40 \\
.058 \\
.039 \\
.77 \\
.36\end{array}$ & $\begin{array}{l}0.32 \\
.16 \\
.029 \\
.027 \\
.29 \\
.13\end{array}$ & $\begin{array}{l}0.30 \\
.20 \\
.030 \\
.022 \\
- \\
-\end{array}$ & $\begin{array}{l}1.2 \\
.70 \\
.111 \\
.098 \\
-\end{array}$ \\
\hline $\begin{array}{l}\text { Nitrite + nitrate, total } \\
\text { Nitrite + nitrate, dissolved } \\
\text { Nitrite, total } \\
\text { Nitrite, dissolved } \\
\text { Nitrate, total } \\
\text { Nitrate, dissolved } C\end{array}$ & $\begin{array}{l}11 \\
11 \\
11 \\
11 \\
- \\
-\end{array}$ & $\begin{array}{l}0.05 \\
D .05 \\
.005 \\
0.004 \\
.04 \\
.05\end{array}$ & $\begin{array}{l}0.06 \\
0.05 \\
.001 \\
0.002 \\
.06 \\
.05\end{array}$ & $\begin{array}{l}<.01 \\
<.01 \\
.004 \\
<.001 \\
- \\
-\end{array}$ & $\begin{array}{l}.15 \\
.14 \\
.007 \\
.008 \\
- \\
-\end{array}$ & $\begin{array}{l}8 \\
8 \\
8 \\
8 \\
- \\
-\end{array}$ & $\begin{array}{l}8.03 \\
B .03 \\
.008 \\
D .003 \\
.02 \\
.03\end{array}$ & $\begin{array}{l}B .05 \\
0.05 \\
.005 \\
0.004 \\
.04 \\
.05\end{array}$ & $\begin{array}{l}<.01 \\
<.01 \\
.003 \\
<.001 \\
- \\
-\end{array}$ & $\begin{array}{l}.15 \\
.14 \\
.016 \\
.012 \\
-\end{array}$ \\
\hline $\begin{array}{l}\text { Nitrogen, total } C \\
\text { Nitrogen, dissolved } C\end{array}$ & - & $\begin{array}{l}.57 \\
.35\end{array}$ & $\begin{array}{l}.35 \\
.15\end{array}$ & - & $\overline{-}$ & $\overline{-}$ & $\begin{array}{l}.86 \\
.43\end{array}$ & $\begin{array}{l}.37 \\
.21\end{array}$ & $\overline{-}$ & - \\
\hline \multicolumn{11}{|c|}{ PHOSPHORUS (mi111grams per Hter as P) } \\
\hline $\begin{array}{l}\text { Phosphorus, total } \\
\text { Orthophosphorua, total } \\
\text { Phosphorus, dissolved } \\
\text { Orthophosphorus dissolved }\end{array}$ & $\begin{array}{l}10 \\
11 \\
10 \\
11\end{array}$ & $\begin{array}{r}0.034 \\
.023 \\
.023 \\
.018\end{array}$ & $\begin{array}{l}0.012 \\
.020 \\
.007 \\
.019\end{array}$ & $\begin{array}{r}0.021 \\
.008 \\
.013 \\
.004\end{array}$ & $\begin{array}{r}0.066 \\
.082 \\
.032 \\
.073\end{array}$ & $\begin{array}{l}8 \\
8 \\
8 \\
8\end{array}$ & $\begin{array}{r}0.072 \\
.017 \\
.012 \\
8.003\end{array}$ & $\begin{array}{r}0.045 \\
.008 \\
.003 \\
8.002\end{array}$ & $\begin{array}{r}0.031 \\
.008 \\
.005 \\
<.001\end{array}$ & $\begin{array}{r}0.161 \\
.028 \\
.016 \\
.008\end{array}$ \\
\hline \multicolumn{11}{|c|}{ OTHER DETERMINATIONS } \\
\hline $\begin{array}{l}\text { Iron, total (ug/L as Fe) } \\
\text { Manganese, total ( } \mathrm{ug} / \mathrm{L} \text { as Mn) } \\
\text { Z1nc, total ( } \mathrm{ug} / \mathrm{L} \text { as } \mathrm{Zn} \text { ) } \\
\text { D18solved sol1ds, ROE at } \\
180^{\circ} \mathrm{C} \text { (mg/L) } \\
\text { Suspended sediment (mg/L) }\end{array}$ & $\begin{array}{l}11 \\
11 \\
11 \\
11\end{array}$ & $\begin{array}{r}630 \\
60 \\
A_{20} \\
81 \\
6\end{array}$ & $\begin{array}{r}280 \\
40 \\
A_{20} \\
7\end{array}$ & $\begin{array}{r}440 \\
20 \\
<10 \\
68\end{array}$ & $\begin{array}{r}1,400 \\
180 \\
80 \\
90\end{array}$ & $\begin{array}{l}8 \\
8 \\
8 \\
8 \\
6\end{array}$ & $\begin{array}{r}1,600 \\
160 \\
30 \\
161 \\
13\end{array}$ & $\begin{array}{r}1,800 \\
110 \\
20 \\
70 \\
12\end{array}$ & $\begin{array}{r}280 \\
50 \\
10 \\
91 \\
4\end{array}$ & $\begin{array}{r}5,200 \\
420 \\
80 \\
290 \\
\\
37\end{array}$ \\
\hline
\end{tabular}

D Value did not differsignificantly when calculated assuming that concentrations below the detection limit ( 1 ) equaled zero and (2) equaled the detection limit.

E statistcal data are for samples collected during March 30-June 4, 1985, only (daca for July 26, 1985, are excluded).

F Median value is shown. The mean was determined to be less satisfactory as a sumary due to a non-normal distribution of measured pH velues. 
TABLE 3.--C'onstituent loads

Site I (Edgewood Creek near Stateline)

[Instantaneous values, expressed in pounds per day]

\begin{tabular}{|c|c|c|c|c|c|c|c|c|c|c|}
\hline Date & $\begin{array}{l}\text { Ammo- } \\
\text { nium, } \\
\text { total } \\
\text { (as } \\
\text { N) }\end{array}$ & $\begin{array}{l}\text { Ammo- } \\
\text { nium, } \\
\text { dis- } \\
\text { solved } \\
\text { (as } N \text { ) }\end{array}$ & $\begin{array}{c}\text { Org- } \\
\text { anic } \\
\text { nitro- } \\
\text { gen, } \\
\text { total } \\
(\text { as } N)\end{array}$ & $\begin{array}{l}\text { Org- } \\
\text { anic } \\
\text { nitro- } \\
\text { gen, } \\
\text { dis- } \\
\text { solved } \\
\text { (as N) }\end{array}$ & $\begin{array}{l}\text { Nit- } \\
\text { rite, } \\
\text { total } \\
\text { (as } \\
N \text { ) }\end{array}$ & $\begin{array}{l}\text { Nit- } \\
\text { rite, } \\
\text { dis- } \\
\text { solved } \\
\text { (as N) }\end{array}$ & $\begin{array}{l}\text { Nit- } \\
\text { rate, } \\
\text { total } \\
\text { (as } \\
\text { N) }\end{array}$ & $\begin{array}{l}\text { Nit- } \\
\text { rate, } \\
\text { dis- } \\
\text { solved } \\
\text { (as N) }\end{array}$ & $\begin{array}{l}\text { Nitro- } \\
\text { gen, } \\
\text { total } \\
\text { (as } \\
\text { N) }\end{array}$ & $\begin{array}{l}\text { Nitro- } \\
\text { gen, } \\
\text { dis- } \\
\text { solved } \\
\text { (as N) }\end{array}$ \\
\hline $8-21-84$ & 0.09 & 0.05 & $<1.9$ & $<2.0$ & 0.05 & 0.07 & 0.41 & 0.31 & $<2.4$ & $<2.4$ \\
\hline $8-21-84$ & .06 & -- & 2.0 & -- & .03 & -- & .54 & - & 2.6 & - \\
\hline $9-6-84$ & .05 & .05 & - & $<1.6$ & .04 & .04 & .35 & $<.09$ & - & $<1.8$ \\
\hline $4-4-85$ & .47 & .43 & 11 & 5.4 & .04 & .06 & 1.6 & 1.6 & 13 & 7.5 \\
\hline $4-15-85$ & 1.0 & 1.0 & 15 & 7.0 & .05 & .11 & 2.7 & 3.0 & 19 & \\
\hline $4-15-85$ & 1.4 & 1.1 & 19 & 16 & .13 & .13 & 4.0 & 4.0 & 24 & 21 \\
\hline $4-23-85$ & .70 & .61 & 6.4 & - & .07 & .04 & 1.2 & 1.2 & 8.4 & - \\
\hline $\begin{array}{l}4-29-85 \\
5-7-85\end{array}$ & .52 & .70 & 7.6 & 4.2 & .05 & .05 & .97 & $\begin{array}{r}.97 \\
52\end{array}$ & 9.1 & 5.9 \\
\hline $5-15-85$ & .44 & - & 1.8 & - & .01 & - & .23 & -- & 2.5 & $<2.7$ \\
\hline $\begin{array}{l}5-23-85 \\
5-28-85\end{array}$ & $\begin{array}{l}.53 \\
.45\end{array}$ & $\begin{array}{l}.52 \\
.40\end{array}$ & $\begin{array}{l}1.7 \\
2.7\end{array}$ & $\begin{array}{l}.57 \\
1.6\end{array}$ & $\begin{array}{l}.03 \\
.01\end{array}$ & $\begin{array}{r}.04 \\
<.01\end{array}$ & $\begin{array}{l}.23 \\
.31\end{array}$ & $\begin{array}{r}.23 \\
<.31\end{array}$ & $\begin{array}{l}2.5 \\
3.5\end{array}$ & $\begin{array}{l}1.4 \\
2.3\end{array}$ \\
\hline $6-4-85$ & .49 & .50 & 3.4 & 2.4 & .03 & .08 & .29 & .19 & 4.2 & 3.2 \\
\hline $7-16-85$ & .16 & .17 & $<.85$ & $<.85$ & .04 & .05 & .30 & .30 & $<1.4$ & $<1.4$ \\
\hline Mean & 0.48 & 0.50 & $A_{5.9}$ & $A_{3.8}$ & 0.04 & $B_{0} .06$ & 0.98 & $A_{1.0}$ & $A_{7.4}$ & $A_{5} .0$ \\
\hline Date & $\begin{array}{l}\text { Phos- } \\
\text { phor- } \\
\text { us, } \\
\text { total } \\
\text { (as } \\
\text { P) }\end{array}$ & $\begin{array}{l}\text { Ortho- } \\
\text { phos- } \\
\text { phor- } \\
\text { us, } \\
\text { total } \\
\text { (as p) }\end{array}$ & $\begin{array}{c}\text { Phos- } \\
\text { phor- } \\
\text { us, } \\
\text { dis- } \\
\text { solved } \\
\text { (as P) }\end{array}$ & $\begin{array}{l}\text { Ortho- } \\
\text { phos- } \\
\text { phor- } \\
\text { us, } \\
\text { dis- } \\
\text { solved } \\
\text { (as P) }\end{array}$ & $\begin{array}{l}\text { Iron, } \\
\text { total } \\
\text { (as } \\
\mathrm{Fe})\end{array}$ & $\begin{array}{l}\text { Mang- } \\
\text { anese, } \\
\text { total } \\
\text { (as } \\
\text { Mn) }\end{array}$ & $\begin{array}{l}\text { Zinc, } \\
\text { total } \\
(\text { as } \\
\mathrm{Zn})\end{array}$ & $\begin{array}{l}\text { Dis- } \\
\text { solved } \\
\text { solids }\end{array}$ & $\begin{array}{l}\text { Sus- } \\
\text { pend- } \\
\text { ed } \\
\text { sed } 1- \\
\text { ment }\end{array}$ & \\
\hline $8-21-84$ & 0.15 & - & 0.11 & - & 8.1 & 0.41 & 0.31 & 850 & 62 & \\
\hline $8-21-84$ & .37 & - & - & - & - & - & - & - & 140 & \\
\hline $9-6-84$ & - & 0.16 & .07 & 0.12 & 5.6 & .17 & $<.09$ & 660 & 52 & \\
\hline $4-4-85$ & - & .25 & .43 & .21 & 29 & 1.6 & .39 & 1,800 & 430 & \\
\hline $4-15-85$ & 1.8 & .40 & .54 & .35 & 68 & 3.8 & .54 & 2,400 & $1 ; 400$ & \\
\hline $4-15-85$ & 2.7 & .54 & .70 & .40 & 130 & 7.0 & 2.3 & 3,000 & 2,700 & \\
\hline $4-23-85$ & .71 & .30 & .39 & .21 & 23 & 1.2 & .18 & 1,600 & 250 & \\
\hline $4-29-85$ & .50 & .29 & .34 & .21 & 15 & .81 & .32 & 1,400 & 470 & \\
\hline $5-7-85$ & .32 & .17 & .27 & - & 11 & .78 & .26 & 1,000 & 100 & \\
\hline $5-15-85$ & .30 & .15 & .23 & .14 & 11 & .57 & .23 & 1,000 & 110 & \\
\hline $5-23-85$ & .78 & .15 & .12 & .15 & 28 & 1.5 & $<.11$ & 1,000 & 230 & \\
\hline $5-28-85$ & .24 & .08 & .15 & .11 & 9.3 & .51 & $<.10$ & 840 & 92 & \\
\hline $6-4-85$ & .30 & .13 & .16 & .16 & 8.1 & .39 & .10 & 830 & 97 & \\
\hline $7-16-85$ & .17 & .06 & .08 & .02 & 3.1 & .20 & .35 & 390 & 30 & \\
\hline Mean & 0.70 & 0.22 & 0.28 & 0.19 & 27 & 1.5 & $A_{0} .40$ & 1,300 & 440 & \\
\hline
\end{tabular}


TABLE 3.--Constituent Loads-Continued

Site 2 (Edgewood Creek below Highway 50)

[Instantaneous values, expressed in pounds per day]

\begin{tabular}{|c|c|c|c|c|c|c|c|c|c|c|}
\hline Date & $\begin{array}{l}\text { Ammo- } \\
\text { nlum, } \\
\text { total } \\
\text { (as } \\
\text { N) }\end{array}$ & $\begin{array}{l}\text { Ammo- } \\
\text { nlum, } \\
\text { d1s- } \\
\text { solved } \\
\text { (as N) }\end{array}$ & $\begin{array}{c}\text { Org- } \\
\text { anic } \\
\text { nitro- } \\
\text { gen, } \\
\text { total } \\
\text { (as } N \text { ) }\end{array}$ & $\begin{array}{l}\text { Org- } \\
\text { anfc } \\
\text { nitro- } \\
\text { gen, } \\
\text { dis- } \\
\text { solved } \\
(\text { as N) }\end{array}$ & $\begin{array}{l}\text { Nit- } \\
\text { rite, } \\
\text { total } \\
\text { (as } \\
\text { N) }\end{array}$ & $\begin{array}{l}\text { Nit- } \\
\text { rite, } \\
\text { dis- } \\
\text { solved } \\
\text { (as N) }\end{array}$ & $\begin{array}{l}\text { N1t- } \\
\text { rate, } \\
\text { total } \\
\text { (as } \\
\text { N) }\end{array}$ & $\begin{array}{l}\text { N1t- } \\
\text { rate, } \\
\text { dis- } \\
\text { solved } \\
\text { (as N) }\end{array}$ & $\begin{array}{l}\text { Nitro- } \\
\text { gen, } \\
\text { total } \\
\text { (as } \\
\text { N) }\end{array}$ & $\begin{array}{l}\text { Nitro- } \\
\text { gen, } \\
\text { dis- } \\
\text { solved } \\
\text { (as N) }\end{array}$ \\
\hline $\begin{array}{l}9-6-84 \\
4-29-85 \\
6-4-85 \\
7-16-85\end{array}$ & $\begin{array}{l}0.21 \\
1.6 \\
1.4 \\
.59\end{array}$ & $\begin{array}{l}0.21 \\
1.7 \\
1.3 \\
.41\end{array}$ & $\begin{array}{l}-- \\
18 \\
6.9 \\
3.0\end{array}$ & $\begin{array}{l}<4.9 \\
10 \\
6.9 \\
<3.2\end{array}$ & $\begin{array}{r}0.10 \\
.12 \\
.08 \\
.12\end{array}$ & $\begin{array}{r}0.13 \\
.12 \\
.19 \\
.09\end{array}$ & $\begin{array}{l}0.26 \\
3.8 \\
.83 \\
.36\end{array}$ & $\begin{array}{c}<0.26 \\
3.8 \\
.83 \\
.36\end{array}$ & $\begin{array}{c}- \\
24 \\
9.2 \\
4.1\end{array}$ & $\begin{array}{l}<5.5 \\
16 \\
9.2 \\
<4.1\end{array}$ \\
\hline Mean & 0.95 & 0.90 & 9.3 & $C_{5.2}$ & 0.10 & 0.13 & 1.3 & $A_{1.3}$ & 12 & $c_{7.5}$ \\
\hline Date & $\begin{array}{l}\text { Phos- } \\
\text { phor- } \\
\text { us, } \\
\text { total } \\
\text { (as } \\
\text { P) }\end{array}$ & $\begin{array}{l}\text { Ortho- } \\
\text { phos- } \\
\text { phor- } \\
\text { us, } \\
\text { total } \\
\text { (as P) }\end{array}$ & $\begin{array}{l}\text { Phos- } \\
\text { phor- } \\
\text { us, } \\
\text { dis- } \\
\text { solved } \\
\text { (as P) }\end{array}$ & $\begin{array}{l}\text { Ortho- } \\
\text { phos- } \\
\text { phor- } \\
\text { us, } \\
\text { dis- } \\
\text { solved } \\
\text { (as P) }\end{array}$ & $\begin{array}{c}\text { Iron, } \\
\text { total } \\
(\mathrm{as} \\
\mathrm{Fe})\end{array}$ & $\begin{array}{l}\text { Mang- } \\
\text { anese, } \\
\text { total } \\
\text { (as } \\
\text { Mn) }\end{array}$ & $\begin{array}{l}\text { Zinc, } \\
\text { total } \\
(a s \\
Z n)\end{array}$ & $\begin{array}{l}\text { Dis- } \\
\text { solved } \\
\text { solids }\end{array}$ & $\begin{array}{l}\text { Sus- } \\
\text { pend- } \\
\text { ed } \\
\text { sed 1- } \\
\text { ment }\end{array}$ & \\
\hline $\begin{array}{l}9-6-84 \\
4-29-85 \\
6-4-85 \\
7-16-85\end{array}$ & $\begin{array}{r}-- \\
0.80 \\
.74 \\
.41\end{array}$ & $\begin{array}{r}0.49 \\
.61 \\
.39 \\
.21\end{array}$ & $\begin{array}{r}0.26 \\
.84 \\
.44 \\
.27\end{array}$ & $\begin{array}{r}0.36 \\
.46 \\
.47 \\
.05\end{array}$ & $\begin{array}{l}14 \\
22 \\
15 \\
11\end{array}$ & $\begin{array}{l}0.52 \\
1.2 \\
1.1 \\
1.1\end{array}$ & $\begin{array}{r}<0.26 \\
.38 \\
<.27 \\
<.18\end{array}$ & $\begin{array}{r}1,500 \\
29,000 \\
2,000 \\
1,200\end{array}$ & $\begin{array}{r}100 \\
460 \\
110 \\
71\end{array}$ & \\
\hline Mean & 0.65 & 0.42 & 0.45 & 0.34 & 16 & 0.98 & $c_{0.18}$ & 1,900 & 180 & \\
\hline
\end{tabular}


TABLE 3.--Constituent Loads--Continued

Site 3 (Edgewood Creek at Lake Tahoe)

[Instantaneous values, expressed In pounds per day]

\begin{tabular}{|c|c|c|c|c|c|c|c|c|c|c|}
\hline Date & $\begin{array}{l}\text { Ammo- } \\
\text { nium, } \\
\text { tota1 } \\
\text { (as } \\
\text { N) }\end{array}$ & $\begin{array}{l}\text { Ammo- } \\
\text { nf um, } \\
\text { dis- } \\
\text { solved } \\
\text { (as N) }\end{array}$ & $\begin{array}{c}\text { Org- } \\
\text { anic } \\
\text { nitro- } \\
\text { gen, } \\
\text { total } \\
(\text { as } N)\end{array}$ & $\begin{array}{c}\text { Org- } \\
\text { anic } \\
\text { nitro- } \\
\text { gen, } \\
\text { dis- } \\
\text { solved } \\
\text { (as N) }\end{array}$ & $\begin{array}{l}\text { Nit- } \\
\text { rite, } \\
\text { total } \\
\text { (as } \\
\text { N) }\end{array}$ & $\begin{array}{l}\text { Nit- } \\
\text { rite, } \\
\text { dis- } \\
\text { solved } \\
\text { (as N) }\end{array}$ & $\begin{array}{l}\text { Nit- } \\
\text { rate, } \\
\text { total } \\
(\text { as } \\
N)\end{array}$ & $\begin{array}{l}\text { Nit- } \\
\text { rate, } \\
\text { dis- } \\
\text { solved } \\
\text { (as } N \text { ) }\end{array}$ & $\begin{array}{l}\text { N1tro- } \\
\text { gen, } \\
\text { total } \\
\text { (as } \\
\text { N) }\end{array}$ & $\begin{array}{l}\text { Nitro- } \\
\text { gen, } \\
\text { dis- } \\
\text { solved } \\
\text { (as N) }\end{array}$ \\
\hline $\begin{array}{l}9-6-84 \\
4-4-85 \\
4-15-85 \\
4-23-85 \\
4-29-85\end{array}$ & $\begin{array}{l}1.8 \\
3.6 \\
3.2 \\
2.4 \\
1.5\end{array}$ & $\begin{array}{l}1.9 \\
3.1 \\
2.7 \\
2.0 \\
1.3\end{array}$ & $\begin{array}{l}-- \\
55 \\
56 \\
20 \\
27\end{array}$ & $\begin{array}{c}<2.3 \\
9.7 \\
21 \\
15 \\
8.1\end{array}$ & $\begin{array}{r}0.10 \\
.45 \\
.24 \\
.22 \\
.12\end{array}$ & $\begin{array}{r}0.10 \\
.32 \\
<.06 \\
.09 \\
.12\end{array}$ & $\begin{array}{c}<0.20 \\
9.1 \\
8.3 \\
3.9 \\
2.2\end{array}$ & $\begin{array}{c}<0.20 \\
9.1 \\
<8.3 \\
3.9 \\
2.2\end{array}$ & $\begin{array}{l}-6 \\
68 \\
68 \\
26 \\
31\end{array}$ & $\begin{array}{l}<4 \cdot 5 \\
22 \\
32 \\
21 \\
12\end{array}$ \\
\hline $\begin{array}{l}5-7-85 \\
5-15-85 \\
5-23-85 \\
5-28-85 \\
6-4-85 \\
7-16-85\end{array}$ & $\begin{array}{c}.80 \\
1.1 \\
.92 \\
1.4 \\
1.1 \\
.20\end{array}$ & $\begin{array}{c}.65 \\
-.58 \\
1.3 \\
1.1 \\
.15\end{array}$ & $\begin{array}{l}5.6 \\
6.7 \\
8.8 \\
7.3 \\
6.2 \\
1.8\end{array}$ & $\begin{array}{l}3.7 \\
9.2 \\
7.3 \\
6.5 \\
1.8\end{array}$ & $\begin{array}{l}.09 \\
.16 \\
.12 \\
.12 \\
.10 \\
.04\end{array}$ & $\begin{array}{l}.04 \\
.21 \\
.05 \\
.09 \\
.20 \\
.04\end{array}$ & $\begin{array}{r}.43 \\
.78 \\
.24 \\
.29 \\
.50 \\
<.06\end{array}$ & $\begin{array}{r}.65 \\
.52 \\
.24 \\
.29 \\
.25 \\
<.06\end{array}$ & $\begin{array}{c}6.9 \\
8.7 \\
10 \\
9.1 \\
7.9 \\
<2.1\end{array}$ & $\begin{array}{c}5.0 \\
8.5 \\
10 \\
9.0 \\
8.0 \\
2.0\end{array}$ \\
\hline Mean & 1.6 & 1.5 & 19 & $A_{8.4}$ & 0.16 & $B_{0} .12$ & $A_{2.4}$ & $A_{1.6}$ & $B_{24}$ & $B_{12}$ \\
\hline Date & $\begin{array}{l}\text { Phos- } \\
\text { phor- } \\
\text { us, } \\
\text { total } \\
\text { (as } \\
\text { P) }\end{array}$ & $\begin{array}{l}\text { Ortho- } \\
\text { phos- } \\
\text { phor- } \\
\text { us, } \\
\text { total } \\
\text { (as P) }\end{array}$ & $\begin{array}{c}\text { Phos- } \\
\text { phor- } \\
\text { us, } \\
\text { d1s- } \\
\text { solved } \\
\text { (as P) }\end{array}$ & $\begin{array}{l}\text { Ortho- } \\
\text { phos- } \\
\text { phor- } \\
\text { us, } \\
\text { dis- } \\
\text { solved } \\
\text { (as P) }\end{array}$ & $\begin{array}{c}\text { Iron, } \\
\text { total } \\
(\mathbf{a s} \\
\mathrm{Fe})\end{array}$ & $\begin{array}{l}\text { Mang- } \\
\text { anese, } \\
\text { tota1 } \\
\text { (as } \\
\text { Mn) }\end{array}$ & $\begin{array}{c}\text { Zinc, } \\
\text { total } \\
(a s \\
\text { Zn) }\end{array}$ & $\begin{array}{l}\text { D1s- } \\
\text { solved } \\
\text { solids }\end{array}$ & $\begin{array}{l}\text { Sus- } \\
\text { pend- } \\
\text { ed } \\
\text { sed1- } \\
\text { ment }\end{array}$ & \\
\hline $\begin{array}{l}9-6-84 \\
4-4-85 \\
4-15-85 \\
4-23-85 \\
4-29-85\end{array}$ & $\begin{array}{l}-- \\
4.3 \\
1.8 \\
1.5 \\
.97\end{array}$ & $\begin{array}{l}1.7 \\
1.8 \\
1.0 \\
.88 \\
.72\end{array}$ & $\begin{array}{l}-\overline{2.1} \\
1.3 \\
1.2 \\
.85\end{array}$ & $\begin{array}{r}1.5 \\
1.4 \\
.83 \\
.66 \\
.53\end{array}$ & $\begin{array}{l}9.0 \\
91 \\
49 \\
29 \\
15\end{array}$ & $\begin{array}{l}0.41 \\
4.5 \\
3.0 \\
2.2 \\
5.6\end{array}$ & $\begin{array}{c}<0.20 \\
1.9 \\
1.8 \\
.44 \\
2.5\end{array}$ & $\begin{array}{l}1,400 \\
5,800 \\
4,600 \\
3,500 \\
2,700\end{array}$ & $\begin{array}{r}82 \\
1,300 \\
480 \\
180 \\
190\end{array}$ & \\
\hline $\begin{array}{l}5-7-85 \\
5-15-85 \\
5-23-85 \\
5-28-85 \\
6-4-85 \\
7-16-85\end{array}$ & $\begin{array}{l}.67 \\
.83 \\
.51 \\
.76 \\
.77 \\
.27\end{array}$ & $\begin{array}{l}.32 \\
.39 \\
.19 \\
.26 \\
.35 \\
.17\end{array}$ & $\begin{array}{l}.48 \\
.67 \\
.36 \\
.38 \\
.42 \\
.21\end{array}$ & $\begin{array}{l}.24 \\
.31 \\
.12 \\
.29 \\
.32 \\
.03\end{array}$ & $\begin{array}{c}10 \\
13 \\
12 \\
15 \\
13 \\
3.8\end{array}$ & $\begin{array}{c}1.1 \\
1.0 \\
.97 \\
1.2 \\
.99 \\
.26\end{array}$ & $\begin{array}{r}.86 \\
.26 \\
.24 \\
<.29 \\
<.25 \\
.13\end{array}$ & $\begin{array}{r}1,800 \\
2,200 \\
2,200 \\
2,300 \\
1,900 \\
480\end{array}$ & $\begin{array}{r}86 \\
100 \\
97 \\
88 \\
150 \\
26\end{array}$ & \\
\hline Mean & 1.2 & 0.71 & 0.80 & 0.57 & 24 & 1.9 & $A_{0} .76$ & 2,600 & 250 & \\
\hline
\end{tabular}

$A$ Mean estimated using the "log-probability regression" method, as discussed by Gilliom and Helsel (1984) and Helsel and Gilliom (1985).

$B$ Value did not differ significantly when calculated assuming that concentrations below the detection limt (1) equaled zero and (2) equaled the detection limit.

$C$ Concentrations below the detection $11 \mathrm{mit}$ were assumed to be equal to one half of the detection limit for purposes of statistical calculations. 